\title{
A MEASUREMENT OF SECONDARY COSMIC MICROWAVE BACKGROUND ANISOTROPIES WITH TWO YEARS OF SOUTH POLE TELESCOPE OBSERVATIONS
}

\author{
C. L. Reichardt ${ }^{1}$, L. Shaw ${ }^{2}$, O. ZahN ${ }^{3}$, K. A. Aird ${ }^{4}$, B. A. Benson ${ }^{5,6}$, L. E. Bleem ${ }^{5,7}$, J. E. Carlstrom ${ }^{5,6,7,8,9}$,

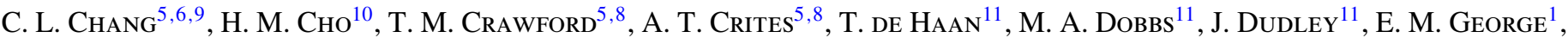 \\ N. W. Halverson ${ }^{12}$, G. P. Holder ${ }^{11}$, W. L. Holzapfel ${ }^{1}$, S. Hoover ${ }^{5,7}$, Z. Hou ${ }^{13}$, J. D. Hrubes ${ }^{4}$, M. JoY ${ }^{14}$, R. KeisleR ${ }^{5,7}$,

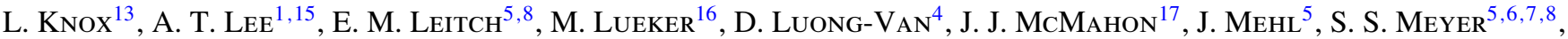

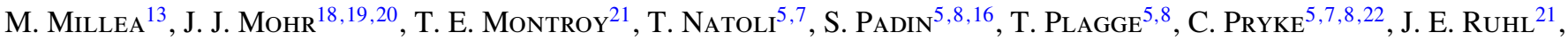 \\ K. K. Schaffer ${ }^{5,6,23}$, E. Shirokoff ${ }^{1}$, H. G. Spieler ${ }^{15}$, Z. Staniszewski ${ }^{21}$, A. A. Stark ${ }^{24}$, K. Story ${ }^{5,7}$, A. van Engelen ${ }^{11}$, \\ K. VANDERLINDE ${ }^{11}$, J. D. Vieira ${ }^{16}$, AND R. Williamson ${ }^{5,8}$ \\ ${ }^{1}$ Department of Physics, University of California, Berkeley, CA 94720, USA; cr@ bolo.berkeley.edu \\ ${ }^{2}$ Department of Physics, Yale University, P.O. Box 208210, New Haven, CT 06520-8120, USA \\ ${ }^{3}$ Berkeley Center for Cosmological Physics, Department of Physics, University of California, \\ and Lawrence Berkeley National Labs, Berkeley, CA 94720, USA \\ ${ }^{4}$ University of Chicago, 5640 South Ellis Avenue, Chicago, IL 60637, USA \\ ${ }^{5}$ Kavli Institute for Cosmological Physics, University of Chicago, 5640 South Ellis Avenue, Chicago, IL 60637, USA \\ ${ }_{7}^{6}$ Enrico Fermi Institute, University of Chicago, 5640 South Ellis Avenue, Chicago, IL 60637, USA \\ ${ }^{7}$ Department of Physics, University of Chicago, 5640 South Ellis Avenue, Chicago, IL 60637, USA \\ ${ }^{8}$ Department of Astronomy and Astrophysics, University of Chicago, 5640 South Ellis Avenue, Chicago, IL 60637, USA \\ ${ }^{9}$ Argonne National Laboratory, 9700 South Cass Avenue, Argonne, IL 60439, USA \\ ${ }^{10}$ NIST Quantum Devices Group, 325 Broadway Mailcode 817.03, Boulder, CO 80305, USA \\ ${ }^{11}$ Department of Physics, McGill University, 3600 Rue University, Montreal, Quebec H3A 2T8, Canada \\ 12 Department of Astrophysical and Planetary Sciences and Department of Physics, University of Colorado, Boulder, CO 80309, USA \\ ${ }^{13}$ Department of Physics, University of California, One Shields Avenue, Davis, CA 95616, USA \\ ${ }^{14}$ Department of Space Science, VP62, NASA Marshall Space Flight Center, Huntsville, AL 35812, USA \\ ${ }^{15}$ Physics Division, Lawrence Berkeley National Laboratory, Berkeley, CA 94720, USA \\ ${ }^{16}$ California Institute of Technology, MS 249-17, 1216 East California Boulevard, Pasadena, CA 91125, USA \\ ${ }^{17}$ Department of Physics, University of Michigan, 450 Church Street, Ann Arbor, MI 48109, USA \\ ${ }^{18}$ Department of Physics, Ludwig-Maximilians-Universität, Scheinerstr. 1, 81679 München, Germany \\ ${ }^{19}$ Excellence Cluster Universe, Boltzmannstr. 2, 85748 Garching, Germany \\ ${ }^{20}$ Max-Planck-Institut für extraterrestrische Physik, Giessenbachstr. 85748 Garching, Germany \\ ${ }^{21}$ Physics Department, Center for Education and Research in Cosmology and Astrophysics, Case Western Reserve University, Cleveland, OH 44106, USA \\ 22 Department of Physics, University of Minnesota, 116 Church Street S.E. Minneapolis, MN 55455, USA \\ ${ }^{23}$ Liberal Arts Department, School of the Art Institute of Chicago, 112 South Michigan Avenue, Chicago, IL 60603, USA \\ ${ }^{24}$ Harvard-Smithsonian Center for Astrophysics, 60 Garden Street, Cambridge, MA 02138, USA \\ Received 2012 April 25; accepted 2012 June 12; published 2012 July 26
}

\begin{abstract}
We present the first three-frequency South Pole Telescope (SPT) cosmic microwave background (CMB) power spectra. The band powers presented here cover angular scales $2000<\ell<9400$ in frequency bands centered at 95 , 150 , and $220 \mathrm{GHz}$. At these frequencies and angular scales, a combination of the primary CMB anisotropy, thermal and kinetic Sunyaev-Zel'dovich (SZ) effects, radio galaxies, and cosmic infrared background (CIB) contributes to the signal. We combine Planck/HFI and SPT data at $220 \mathrm{GHz}$ to constrain the amplitude and shape of the CIB power spectrum and find strong evidence for nonlinear clustering. We explore the SZ results using a variety of cosmological models for the CMB and CIB anisotropies and find them to be robust with one exception: allowing for spatial correlations between the thermal SZ effect and CIB significantly degrades the SZ constraints. Neglecting this potential correlation, we find the thermal SZ power at $150 \mathrm{GHz}$ and $\ell=3000$ to be $3.65 \pm 0.69 \mu \mathrm{K}^{2}$, and set an upper limit on the kinetic SZ power to be less than $2.8 \mu \mathrm{K}^{2}$ at $95 \%$ confidence. When a correlation between the thermal SZ and CIB is allowed, we constrain a linear combination of thermal and kinetic SZ power: $D_{3000}^{\mathrm{tSZ}}+0.5 D_{3000}^{\mathrm{kSZ}}=4.60 \pm 0.63 \mu \mathrm{K}^{2}$, consistent with earlier measurements. We use the measured thermal SZ power and an analytic, thermal SZ model calibrated with simulations to determine $\sigma_{8}=0.807 \pm 0.016$. Modeling uncertainties involving the astrophysics of the intracluster medium rather than the statistical uncertainty in the measured band powers are the dominant source of uncertainty on $\sigma_{8}$. We also place an upper limit on the kinetic SZ power produced by patchy reionization; a companion paper uses these limits to constrain the reionization history of the universe.
\end{abstract}

Key words: cosmic background radiation - cosmological parameters - cosmology: observations diffuse radiation - large-scale structure of universe

Online-only material: color figures

\section{INTRODUCTION}

The current generation of ground-based cosmic microwave background (CMB) experiments enables researchers to probe, for the first time, the power spectra of the thermal and kinetic Sunyaev-Zel'dovich (SZ) effects (Sunyaev \& Zel'dovich 1972; Sunyaev \& Zeldovich 1980). The South Pole Telescope (SPT; Carlstrom et al. 2011) and the Atacama Cosmology Telescope 
(ACT; Fowler et al. 2007) have sufficient sensitivity, angular resolution, and frequency coverage to measure the power spectrum of the thermal SZ (tSZ) effect and to place increasingly strong upper limits on contributions from the kinetic SZ (kSZ) effect (Lueker et al. 2010; Shirokoff et al. 2011; Das et al. 2011). These secondary temperature anisotropies in the CMB arise when $\mathrm{CMB}$ photons are scattered by the inhomogeneous distribution of free electrons well after recombination. As such, they depend sensitively on both the growth of structure and the details of reionization.

The tSZ effect occurs when CMB photons inverse Compton scatter off hot electrons, predominantly those gravitationally bound in the potential wells of galaxy clusters. The scattering tends to increase the photon energy, distorting the CMB blackbody spectrum with a loss of photons below approximately $217 \mathrm{GHz}$ and an excess at higher frequencies. The kSZ effect occurs when CMB photons are Doppler shifted by the bulk velocity of electrons in intervening ionized gas. The kSZ effect changes the apparent $\mathrm{CMB}$ temperature while maintaining the blackbody spectrum. We can discriminate between the two effects based on their frequency dependence.

The anisotropy power due to the tSZ effect is highly sensitive to the normalization of the matter power spectrum, commonly parameterized by the rms of the $z=0$ mass distribution on $8 \mathrm{Mpc}^{-1}$ scales, $\sigma_{8}$ (Komatsu \& Seljak 2002). As a measure of structure growth, the tSZ power spectrum can provide independent constraints on cosmological parameters and improve the precision with which they are determined. For instance, a measurement of the tSZ power spectrum can be used to determine the sum of the neutrino masses by breaking the degeneracy between $\sigma_{8}$ and neutrino mass that exists with the $\mathrm{CMB}$ data alone. The tSZ power spectrum can also be used to constrain non-standard cosmological models, for example, placing limits on the range of allowed early dark energy models (Alam et al. 2011).

The tSZ power spectrum is challenging to model accurately because it includes significant contributions from galaxy clusters spanning a wide mass and redshift range. Modeling uncertainties arise from both non-gravitational heating effects in low-mass clusters and the limited body of observational data on highredshift clusters. Recent models that vary in their treatment of cluster gas physics differ in amplitude by up to $50 \%$ for a given cosmology (Shaw et al. 2010; Trac et al. 2011; Battaglia et al. 2010).

The post-reionization $\mathrm{kSZ}$ power spectrum is expected to be easier to model than the tSZ spectrum. As it is not weighted by the gas temperature, the $\mathrm{kSZ}$ power spectrum depends less on nonlinear effects that complicate models of the tSZ signal. The total kSZ power spectrum does, however, depend strongly on the details of reionization, which are not yet well understood. In the standard picture of reionization, ionized bubbles form around the first stars or quasars. These bubbles eventually overlap and merge, leading to a fully ionized universe. The ionized bubbles impart a Doppler shift on scattered CMB photons proportional to their line-of-sight velocity. This so-called patchy reionization $\mathrm{kSZ}$ signal depends on the duration of reionization as well as the distribution of bubble sizes (Gruzinov \& Hu 1998; Knox et al. 1998). Detection of the kSZ power spectrum or even an upper limit on its amplitude would lead to interesting constraints on the epoch of reionization (Zahn et al. 2005).

The first detection of the combined tSZ and kSZ spectra was presented by Lueker et al. (2010, hereafter L10) using data from the SPT. Since then, improved measurements of the power spectra have been reported by Shirokoff et al. (2011, hereafter S11) based on 150 and $220 \mathrm{GHz}$ data for $200 \mathrm{deg}^{2}$ of sky. S11 report a $4.5 \sigma$ detection of SZ power at $152 \mathrm{GHz}$ at a multipole of $\ell=3000$ with an amplitude of $D_{3000}^{\mathrm{tSZ}}+0.5 D_{3000}^{\mathrm{kSZ}}=$ $4.5 \pm 1.0 \mu \mathrm{K}^{2} .{ }^{25}$ The $95 \%$ confidence upper limits on each component were $D_{3000}^{\mathrm{tSZ}}<5.3 \mu \mathrm{K}^{2}$ and $D_{3000}^{\mathrm{kSZ}}<6.5 \mu \mathrm{K}^{2}$. The measured tSZ power is less than predicted by some models, suggesting that either these models overpredict the tSZ power or $\sigma_{8}$ is significantly less than the Wilkinson Microwave Anisotropy Probe (WMAP) preferred value of $\sim 0.8$ (Larson et al. 2011).

The ACT collaboration has also measured the high- $\ell$ power spectrum (Das et al. 2011). Dunkley et al. (2011) use the Das et al. (2011) bandpowers to measure the sum of the tSZ and kSZ power at $\ell=3000$ and $148 \mathrm{GHz}$ to be $6.8 \pm 2.9 \mu \mathrm{K}^{2}$. The SPT and ACT bandpowers and resulting constraints on SZ power are consistent within the reported uncertainties.

The sky power at millimeter wavelengths and arcminute scales is dominated by the signal from bright synchrotron sources, with additional contributions from nearby infrared galaxies and a population of high-redshift, gravitationally lensed, dusty, star-forming galaxies (DSFGs; Vieira et al. 2010). After these identifiable sources are masked, the power spectrum is dominated by the cosmic infrared background (CIB), the bulk of which is contributed by unlensed DSFGs below the detection threshold of instruments like SPT and ACT (Lagache et al. 2005). These DSFGs are important both as a foreground for CMB experiments and as a tool to understand the history of star and galaxy formation (e.g., Bond et al. 1986, 1991; Knox et al. 2001; Lagache et al. 2005). The power from DSFGs has both Poisson and clustered components, with distinct angular scale dependencies. Measurements of CIB power at multiple millimeter wavelengths can be combined to constrain the spectral index of the CIB anisotropy. Note that this depends on both the spectral energy distribution of individual DSFGs and their redshift distribution. S11 constrained the spectral index of the DSFGs between 150 and $220 \mathrm{GHz}$ to be $\alpha=3.58 \pm 0.09$. Dunkley et al. (2011) also detected significant power attributed to clustered DSFGs in the ACT data and found a preferred DSFG spectral index of $3.69 \pm 0.14$, consistent with the $S 11$ results. The Planck Collaboration et al. (2011) combined the Planck/ HFI CIB bandpowers with the S11 bandpowers to show that the combined data set is inconsistent with only the Poisson and linear clustering terms-nonlinear clustering is required.

This is the fourth SPT power spectrum analysis focused on CIB and secondary CMB anisotropies. Here, we improve upon the previous work by L10, Hall et al. (2010), and S11 in two ways. First, we are analyzing three-frequency data (with six cross-spectra) where earlier analyses used two frequency data with three cross-spectra. The additional frequency information allows the separation of CIB and SZ components. Second, we are analyzing four times more sky area $\left(800 \mathrm{deg}^{2}\right)$ with the concomitant reduction in bandpower uncertainties. A companion paper, Zahn et al. (2011, Z11), interprets the derived kSZ constraints in light of the epoch of reionization.

The paper is organized as follows. In Section 2, we present the analysis and the data to which it is applied. We examine the results of tests for systematic errors in the data in Section 3. The resulting power spectrum is presented in Section 4. The

\footnotetext{
25 Throughout this work, the unit $\mathrm{K}$ refers to equivalent fluctuations in the CMB temperature, i.e., the temperature fluctuation of a $2.73 \mathrm{~K}$ blackbody that would be required to produce the same power fluctuation. The flux conversion factor is given by the derivative of the blackbody spectrum, $(d B / d T)$, evaluated at $2.73 \mathrm{~K}$.
} 
cosmological modeling is discussed in Section 5 and basic results in Section 6. We explore constraints on the tSZ effect in Section 7, on the kSZ effect in Section 8, and on the CIB in Section 9. We conclude in Section 10.

\section{DATA AND ANALYSIS}

We present bandpowers from $800 \mathrm{deg}^{2}$ of sky observed with the SPT at 95, 150, and $220 \mathrm{GHz}$. Bandpowers are estimated using a pseudo- $C_{\ell}$ cross-spectrum method. We calibrate the data by comparing the power spectrum to seven-year WMAP (WMAP7; Larson et al. 2011) bandpowers.

\subsection{Data}

The $800 \mathrm{deg}^{2}$ of sky analyzed in this work were observed by SPT during the 2008 and 2009 Austral winters. The specific field locations and extents can be found in Table 1 of Keisler et al. (2011, K11). SPT is an off-axis Gregorian telescope with a $10 \mathrm{~m}$ diameter primary mirror located at the South Pole. The receiver is equipped with 960 horn-coupled spiderweb bolometers with superconducting transition edge sensors. The telescope and receiver are discussed in more detail in Ruhl et al. (2004), Padin et al. (2008), and Carlstrom et al. (2011).

Each field was observed to an approximate depth of $18 \mu \mathrm{K}$ $\operatorname{arcmin}$ at $150 \mathrm{GHz}$. The noise levels at 95 and $220 \mathrm{GHz}$ vary significantly between the two years as the focal plane was refurbished to maximize sensitivity to the tSZ effect before the 2009 observing season. This refurbishment added science-quality $95 \mathrm{GHz}$ detectors and removed half the $220 \mathrm{GHz}$ detectors. As a result, the 2009 data are comparatively shallower at $220 \mathrm{GHz}$, but much deeper at $95 \mathrm{GHz}$. The 2009 sky coverage accounts for approximately $75 \%$ of the total sky used in this analysis. When calculating the power spectra, the 2009 data have a statistical weight of $100 \%$ at $95 \mathrm{GHz}, 73 \%$ at $150 \mathrm{GHz}$, and $43 \%$ at $220 \mathrm{GHz}$.

\subsection{Beams and Calibration}

The SPT beams are measured for each year using a combination of bright point sources in each field, Venus, and Jupiter as described in S11. The main lobes of the SPT beam at 95, 150 , and $220 \mathrm{GHz}$ are well represented by $1.7,1.2$, and 1.0 FWHM Gaussians. As in S11, we calculate the principal components of the full covariance matrix of this measurement and find that three eigenvectors are adequate to represent $>99 \%$ of the covariance. We treat the eigenvectors from different years as independent. We have tested the opposite extreme, treating them as completely correlated, and have found minimal impact on the science results. The beam uncertainty is included in the bandpower covariance matrix as described in Section 2.3.4.

The observation-to-observation relative calibrations of the time-ordered data (TOD) are determined from repeated measurements of a galactic H II region, RCW38. As in K11, the absolute calibration at each frequency is determined by comparing the SPT and WMAP7 bandpowers over the multipole range $\ell \in[650,1000]$. We use the same $\ell$-bins for each experiment: seven bins with $\Delta \ell=50$. For the absolute calibration, we do not weight individual modes within each bin (as we will do in Section 2.3). A uniform weighting is used because these large angular scales are dominated by sample variance rather than instrumental and atmospheric noise. This calibration method is model-independent, requiring only that the $\mathrm{CMB}$ power in the SPT fields is statistically representative of the all-sky power. We estimate the uncertainty in the SPT power calibration at 95, 150, and $220 \mathrm{GHz}$ to be $3.5 \%, 3.2 \%$, and $4.8 \%$, respectively. These uncertainties are highly correlated because the main sources of error, WMAP7 bandpower errors, and SPT sample variance are nearly identical between bands.

\subsection{Bandpower Estimation}

We use a pseudo- $C_{\ell}$ method to estimate the bandpowers (Hivon et al. 2002). In pseudo- $C_{\ell}$ methods, bandpowers are estimated directly from the spherical harmonic transform of the map after correcting for effects such as TOD filtering, beams, and finite sky coverage. We process the data using a cross-spectrum-based analysis (Polenta et al. 2005; Tristram et al. 2005) to eliminate noise bias. Our scan strategy produces of the order of 100 complete but noisy maps of each field and so is ideally suited to a cross-spectrum analysis. We use a flat-sky approximation. Before Fourier transforming, each map is apodized by a window designed to mask point sources detected at greater than $>5 \sigma(6.4 \mathrm{mJy})$ at $150 \mathrm{GHz}$, and to avoid sharp edges at the map borders. Beam and filtering effects are corrected for according to the formalism in the MASTER algorithm (Hivon et al. 2002). We report the bandpowers in terms of $\mathcal{D}_{\ell}$, where

$$
\mathcal{D}_{\ell}=\frac{\ell(\ell+1)}{2 \pi} C_{\ell} .
$$

Details of the bandpower estimator have been presented in previous SPT papers (L10; S11; K11). In the following sections, we will only note differences between the current analysis and what was done by S11.

\subsubsection{Simulations}

Simulations are used to estimate the transfer function and sample variance. For each field, we construct simulated observations of 100 sky realizations that have been smoothed by the appropriate beam. The sky realizations are sampled using the SPT pointing information, filtered identically to the real data, and processed into maps.

Each simulated sky is a Gaussian realization of the sum of the best-fit lensed WMAP7 $\Lambda \mathrm{CDM}$ primary CMB model, a kSZ model, and point-source contributions. Note that this approach neglects non-Gaussianity in the $\mathrm{kSZ}$ and radio source contributions and may slightly underestimate the sample variance as a result. Millea et al. (2012) argue that this is negligible for SPT since the non-Gaussian signals are small and, on the relevant angular scales, the bandpower uncertainties are dominated by instrumental noise. The $\mathrm{kSZ}$ power spectrum is taken from the Sehgal et al. (2010) simulations and has an amplitude of $2.05 \mu \mathrm{K}^{2}$ at $\ell=3000$. We include both Poisson and clustered point sources. The Poisson contribution reflects both radio source and DSFG populations. The amplitude of the radio source term is set by the de Zotti et al. (2005) model source counts to an amplitude $D_{3000}^{r}=1.28 \mu \mathrm{K}^{2}$ at $150 \mathrm{GHz}$ with an assumed spectral index of $\alpha_{r}=-0.6$. The amplitude of the Poisson DSFG term at $150 \mathrm{GHz}$ is $D_{3000}^{p}=7.7 \mu \mathrm{K}^{2}$. Finally, the clustered DSFG component is modeled by a $D_{\ell} \propto \ell$ term normalized to $D_{3000}^{p}=5.9 \mu \mathrm{K}^{2}$ at $150 \mathrm{GHz}$. The DSFG terms have an assumed spectral index of 3.6. The amplitude of each component was selected to be consistent with the S11 bandpowers.

\subsubsection{Covariance Estimation and Conditioning}

The bandpower covariance matrix includes two terms: sample variance and instrumental noise variance. The sample variance 
Table 1

Bandpowers

\begin{tabular}{|c|c|c|c|c|c|c|c|}
\hline \multirow[t]{2}{*}{$\ell$ Range } & \multirow[t]{2}{*}{$\ell_{\mathrm{eff}}$} & \multicolumn{2}{|c|}{$95 \mathrm{GHz}$} & \multicolumn{2}{|c|}{$150 \mathrm{GHz}$} & \multicolumn{2}{|c|}{$220 \mathrm{GHz}$} \\
\hline & & $\hat{D}\left(\mu \mathrm{K}^{2}\right)$ & $\sigma\left(\mu \mathrm{K}^{2}\right)$ & $\hat{D}\left(\mu \mathrm{K}^{2}\right)$ & $\sigma\left(\mu \mathrm{K}^{2}\right)$ & $\hat{D}\left(\mu \mathrm{K}^{2}\right)$ & $\sigma\left(\mu \mathrm{K}^{2}\right)$ \\
\hline 2001-2200 & 2073 & 217.4 & 9.1 & 212.9 & 4.8 & 285.2 & 14.3 \\
\hline $2201-2400$ & 2274 & 138.4 & 6.4 & 130.1 & 3.1 & 203.0 & 11.3 \\
\hline $2401-2600$ & 2479 & 107.5 & 5.8 & 97.4 & 2.5 & 188.3 & 10.8 \\
\hline 2601-2800 & 2685 & 75.1 & 5.7 & 73.9 & 2.0 & 171.7 & 10.5 \\
\hline 2801-3000 & 2883 & 51.9 & 4.5 & 55.1 & 1.7 & 175.6 & 10.5 \\
\hline $3001-3400$ & 3190 & 47.5 & 4.3 & 41.5 & 1.2 & 171.2 & 9.1 \\
\hline $3401-3800$ & 3594 & 32.8 & 5.3 & 32.5 & 1.1 & 179.8 & 10.3 \\
\hline $3801-4200$ & 3996 & 35.5 & 6.3 & 32.5 & 1.3 & 208.2 & 12.4 \\
\hline $4201-4600$ & 4400 & 37.2 & 8.9 & 35.9 & 1.5 & 224.0 & 13.9 \\
\hline $4601-5000$ & 4803 & 14.8 & 12.1 & 37.5 & 1.8 & 273.3 & 17.0 \\
\hline $5001-5800$ & 5406 & 31.3 & 13.4 & 45.4 & 1.8 & 341.8 & 18.3 \\
\hline $5801-6600$ & 6209 & 38.6 & 22.4 & 57.0 & 2.5 & 422.0 & 23.3 \\
\hline $6601-7400$ & 7012 & 26.8 & 41.7 & 70.6 & 3.6 & 455.1 & 27.9 \\
\hline $7401-8400$ & 7917 & -16.3 & 78.3 & 87.7 & 5.0 & 614.0 & 39.8 \\
\hline \multirow[t]{2}{*}{$8401-9400$} & 8924 & 34.7 & 156.0 & 96.2 & 7.3 & 792.5 & 62.1 \\
\hline & & \multicolumn{2}{|c|}{$95 \times 150 \mathrm{GHz}$} & \multicolumn{2}{|c|}{$95 \times 220 \mathrm{GHz}$} & \multicolumn{2}{|c|}{$150 \times 220 \mathrm{GHz}$} \\
\hline 2001-2200 & 2073 & 207.6 & 5.8 & 207.2 & 9.8 & 226.0 & 7.1 \\
\hline 2201-2400 & 2274 & 129.6 & 3.8 & 131.7 & 7.1 & 148.6 & 4.8 \\
\hline $2401-2600$ & 2479 & 94.0 & 3.1 & 97.7 & 6.3 & 112.8 & 3.9 \\
\hline 2601-2800 & 2685 & 72.1 & 2.5 & 80.7 & 6.3 & 97.4 & 3.5 \\
\hline 2801-3000 & 2883 & 49.9 & 2.1 & 59.5 & 5.6 & 78.6 & 3.2 \\
\hline $3001-3400$ & 3190 & 37.9 & 1.6 & 39.3 & 4.5 & 69.2 & 2.4 \\
\hline $3401-3800$ & 3594 & 22.9 & 1.6 & 22.4 & 5.5 & 64.8 & 2.4 \\
\hline $3801-4200$ & 3996 & 25.0 & 1.9 & 38.3 & 6.6 & 72.4 & 2.8 \\
\hline $4201-4600$ & 4400 & 25.5 & 2.2 & 23.3 & 8.2 & 77.6 & 3.2 \\
\hline $4601-5000$ & 4803 & 24.1 & 2.9 & 42.0 & 10.7 & 94.5 & 4.0 \\
\hline $5001-5800$ & 5406 & 31.4 & 3.2 & 53.6 & 10.8 & 111.4 & 4.2 \\
\hline 5801-6600 & 6209 & 38.5 & 4.9 & 51.9 & 17.0 & 139.3 & 5.4 \\
\hline $6601-7400$ & 7012 & 48.1 & 7.8 & 40.0 & 26.3 & 169.9 & 7.4 \\
\hline 7401-8400 & 7917 & 57.0 & 13.1 & 122.5 & 39.9 & 211.2 & 9.6 \\
\hline 8401-9400 & 8924 & 83.9 & 24.4 & 109.3 & 79.8 & 282.0 & 15.6 \\
\hline
\end{tabular}

Notes. $\ell$-band multipole range, weighted multipole value $\ell_{\text {eff }}$, bandpower $\hat{D}$, and bandpower uncertainty $\sigma$ for the six auto and cross-spectra of the $95 \mathrm{GHz}, 150 \mathrm{GHz}$, and $220 \mathrm{GHz}$ maps. The quoted uncertainties are based on the diagonal elements of the covariance matrix, which includes the instrumental noise, Gaussian sample variance, and beam uncertainties. The sample variance of the tSZ effect and calibration uncertainty are not included. Calibration uncertainties are quoted in Section 2.2. Point sources with flux $>6.4 \mathrm{mJy}$ at $150 \mathrm{GHz}$ have been masked out at all frequencies in this analysis. This flux cut substantially reduces the contribution of radio sources to the bandpowers, although DSFGs below this threshold contribute significantly to the bandpowers.

is estimated with the signal-only Monte Carlo bandpowers. The instrumental noise variance is calculated from the distribution of the cross-spectrum bandpowers $D_{b}^{v_{i} \times v_{j}, A B}$ between observations $\mathrm{A}$ and $\mathrm{B}$, and frequencies $v_{i}$ and $v_{j}$, as described in L10. We expect some statistical uncertainty of the form

$$
\left\langle\left(\mathbf{C}_{b b^{\prime}}-\left\langle\mathbf{C}_{b b^{\prime}}\right\rangle\right)^{2}\right\rangle=\frac{\mathbf{C}_{b b^{\prime}}^{2}+\mathbf{C}_{b b} \mathbf{C}_{b^{\prime} b^{\prime}}}{n_{\mathrm{obs}}}
$$

in the estimated bandpower covariance matrix. Here, $n_{\mathrm{obs}}$ is the number of observations that go into the covariance estimate. This uncertainty on the covariance is expected to be significantly higher than the true covariance for almost all off-diagonal terms due to the dependence of the uncertainty on the (large) diagonal covariances. We reduce the impact of this uncertainty by "conditioning" the covariance matrix.

How we condition the covariance matrix is determined by the form we expect it to assume. The covariance matrix can be viewed as a set of 36 square blocks, with the six on-diagonal blocks corresponding to the covariances of $95 \times 95,95 \times$
$150, \ldots, 220 \times 220 \mathrm{GHz}$ spectra. The bandpowers reported in Table 1 are obtained by first computing power spectra and covariance matrices for bins of width $\Delta \ell=200$ with a total of 37 initial $\ell$-bins, thus each of these blocks is a $37 \times 37$ matrix. The shape of the correlation matrix in each of these blocks is expected to be the same, as it is set by the apodization window. As a first step to conditioning the covariance matrix, we calculate the correlation matrices for the six on-diagonal blocks and average all off-diagonal elements at a fixed separation from the diagonal in each block,

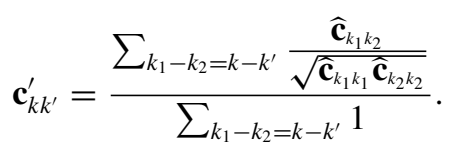

We set the correlation to zero at distances $\ell>400$ where we find no evidence for correlations. We have tested that varying this condition by a factor of two in either direction does not affect the cosmological constraints. This averaged correlation matrix is then applied to all the blocks. 
The covariance matrix includes an estimate of the signal and, if both bandpowers share a common map, the noise variance. To illustrate the latter condition, instrumental noise is included in the blocks corresponding to $(150 \times 150,150 \times 150)$ and $(95 \times 150,150 \times 220)$ covariances, but not the covariance between the $150 \times 150$ and $220 \times 220$ bandpowers. Given the map filtering, we neither expect nor observe correlated noise between different frequencies in the signal band.

We impose one final condition on the estimated covariances of off-diagonal blocks for which the instrumental noise term is included, e.g., the $(95 \times 150,150 \times 220)$ covariance. In these blocks, the noise on the covariance estimate for an $\ell$-bin can be large compared to the true covariance on small angular scales. As a result, we fit an approximate template based on the measured beam shapes from $\ell=2000$ to 4400 and extrapolate this template to estimate the diagonal elements of the covariance matrix at $\ell>4400$. We have tested zeroing these elements of the covariance matrix instead and found that it does not affect the cosmological constraints.

\subsubsection{Field Weighting}

We construct a diagonal weight matrix for each field $i$, $w_{b b}^{i}=1 / C_{b b}^{i}$. We smooth the weights with $\Delta \ell=1000$ at $\ell<3000$ and $\Delta \ell=2600$ on smaller scales to deal with uncertainty in the covariance estimate since we expect the optimal weights to vary slowly with angular scale.

The combined bandpowers are calculated from the individualfield bandpowers according to

$$
D_{b}=\sum_{i} D_{b}^{i} w_{b b}^{i}
$$

where

$$
\sum_{i} w_{b b}^{i}=1
$$

The covariance matrix is combined likewise according to

$$
\mathbf{C}_{b b^{\prime}}=\sum_{i} w_{b b}^{i} \mathbf{C}_{b b^{\prime}}^{i} w_{b^{\prime} b^{\prime}}^{i}
$$

\subsubsection{Beam Uncertainties}

The procedure for estimating the beams and beam uncertainties is described in Section 2.2; here we discuss how the beam uncertainties are included in the cosmological analysis. In S11, three parameters per frequency band describing the beam uncertainties were marginalized over in the Monte Carlo Markov chain (MCMC) with a Gaussian prior. In this work, we instead follow the treatment laid out in K11 and include the beam uncertainties in the bandpower covariance matrix.

Following K11, we estimate the "beam correlation" matrix for each year:

$$
\boldsymbol{\rho}_{b b^{\prime}}^{\text {beam }}=\left(\frac{\delta D_{b}}{D_{b}}\right)\left(\frac{\delta D_{b^{\prime}}}{D_{b^{\prime}}}\right)
$$

where

$$
\frac{\delta D_{b}}{D_{b}}=1-\left(1+\frac{\delta B_{b}}{B_{b}}\right)^{-2},
$$

and $B_{b}$ is the Fourier transform of the beam map averaged across bin $b$.
We combine the correlation matrix for each year according to the yearly weights. The correlation matrix is translated into a covariance matrix by multiplying by the measured bandpowers:

$$
\mathbf{C}_{b b^{\prime}}^{\text {beam }}=\boldsymbol{\rho}_{b b^{\prime}}^{\text {beam }} D_{b} D_{b^{\prime}} .
$$

This beam covariance is added to the bandpower covariance matrix due to sample variance and instrumental noise.

\section{JACKKNIFE TESTS}

We test for systematic errors using a suite of "jackknife" tests. A jackknife test consists of dividing the data into two halves and differencing them to remove true astrophysical signals. The halves are chosen to maximize the signal in the differenced spectrum due to a potential systematic bias. We then compare the differenced spectrum to the (nearly) zero expectation in the absence of a systematic error. To implement jackknife tests in the cross-spectrum framework, we calculate the crossspectrum of differenced pairs of observations. More details on the implementation can be found in L10 and S11. We look for systematic effects in the following dimensions.

1. Scan direction. We difference the data based on whether the telescope is scanning to the left or right. This test is sensitive to direction- or turnaround-dependent effects, such as detector time constants, or microphonics induced by telescope acceleration.

2. Azimuthal range. We difference the data based on the azimuth at which it was observed. This is primarily a test for ground pickup. As discussed in S11, we select the azimuth ranges for the difference set based on the magnitude of observed ground emission on several degree scales.

3. Time. We have two time-dependent tests. First, we compare the first and second halves of the data for each field. Second, we difference the bandpowers for 2008 from those of 2009. Unlike the other jackknife tests, this yeardifference test is applied only to 150 and $220 \mathrm{GHz}$; there are no $95 \mathrm{GHz}$ data from 2008. This year difference is also implemented differently than the other jackknife tests. We calculate power spectra for each year and difference the bandpowers. Notably, this means that the test is less sensitive since the error bars must include sample variance and beam uncertainties. These two tests are sensitive to long-timescale drifts in the calibration, pointing, detector properties, or any other time-varying effect.

We find the results of each test to be consistent with zero. The results are plotted in Figure 1. Combining all three frequencies, the probabilities to exceed (PTE) for the "first-half-secondhalf," "left-right," and "azimuth-split" jackknives are 0.90, 0.21, and 0.84 , respectively. The PTE of the observed $\chi^{2}$ 's is 0.50 for the three tests at $95 \mathrm{GHz}$. For the four tests at $150 \mathrm{GHz}$ and $220 \mathrm{GHz}$, the PTE are 0.48 and 0.61 , respectively. There is no evidence for systematic effects in the SPT data.

\section{BANDPOWERS}

Applying the analysis described in Section 2.3 to the $800 \mathrm{deg}^{2}$ of sky observed with SPT in 2008 and 2009 leads to the bandpowers shown in Figure 2 and tabulated in Table 1. The bandpowers, covariance matrix, and window functions are available for download on the SPT Web site. ${ }^{26}$

\footnotetext{
26 http://pole.uchicago.edu/public/data/reichardt11/
} 


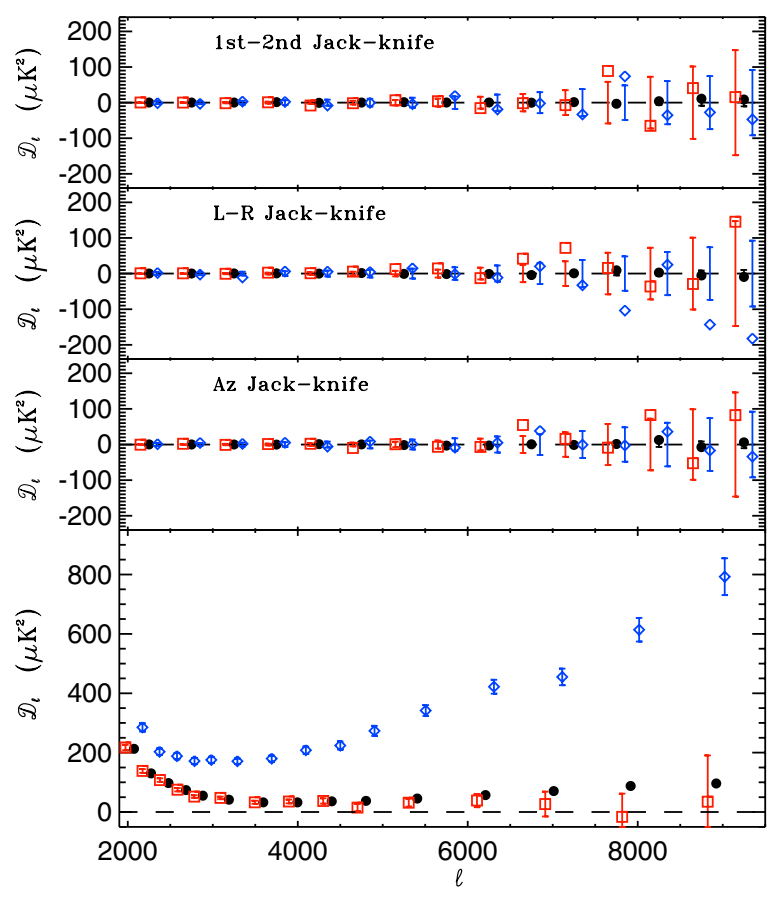

Figure 1. Results of the jackknife tests applied to the SPT data. Red squares denote the $95 \mathrm{GHz}$ bandpowers, black circles mark the $150 \mathrm{GHz}$ bandpowers, and the $220 \mathrm{GHz}$ bandpowers are plotted with blue diamonds. Note that the $150 \mathrm{GHz}$ uncertainties are smaller than the symbols. The top panel displays the results of the "first-half-second-half" jackknife. The PTE to exceed the measured $\chi^{2}$ is 0.90 . The "left-right" jackknife is shown in the second panel and has a PTE of 0.21. The "azimuth-split" jackknife is plotted in the third panel and has a PTE of 0.84. Finally, the auto-spectra of the three frequencies are plotted in the bottom panel for comparison.

(A color version of this figure is available in the online journal.)

A combination of the primary CMB anisotropy, foregrounds, and secondary SZ anisotropies is detected at high $\mathrm{S} / \mathrm{N}$ from $\ell=2000$ to 9400 in all three frequency bands (see Figure 3). The largest signal in the maps is the primary CMB anisotropy; at $\ell<3000$ the bandpowers trace out the Silk damping tail of the CMB power spectrum. The next largest signal is the CIB anisotropy which becomes dominant around $\ell=3000$. At high multipoles, the bandpowers follow the expected $\ell^{2}$ form for Poisson power from point sources. The spectral index of the point-source contribution is $\alpha \simeq 3.5$ between 150 and $220 \mathrm{GHz}$, characteristic of the Rayleigh-Jeans tail of a dust spectrum. Poisson fluctuations of radio sources become important as well at $95 \mathrm{GHz}$. Over these angular scales, the CMB and Poisson fluctuation power from point sources account for approximately $80 \%$ of the power in all frequency bands. We also detect the tSZ effect and clustering component of the CIB at high significance.

\section{COSMOLOGICAL MODELING}

We fit the SPT bandpowers to a model including lensed primary CMB anisotropy, secondary tSZ and kSZ anisotropies, and foregrounds. As stated in the previous section, the CIB is the most important foreground and it is composed primarily of emission from a population of DSFGs. The power spectrum of these DSFGs can be divided into "Poisson" and "clustered" components. Radio sources are also important in the lower frequency bands. Finally, we include a template for the galactic cirrus emission, which is negligible by virtue of the selected sky coverage.

Parameter constraints are calculated using the publicly available CosmoMC ${ }^{27}$ package (Lewis \& Bridle 2002). We have added two modules to handle the high- $\ell$ data: one to model the foregrounds and secondary anisotropies and one to calculate the SPT and Planck/HFI likelihood functions. The code is a modified and expanded version of the modules discussed in Millea et al. (2012) and used in S11. These modules and instructions for compiling them are available at the SPT Web site. $^{28}$

We include measurements of the primary CMB anisotropy, baryon acoustic oscillations (BAO), and Hubble constant $\left(H_{0}\right)$ in all cosmological constraints presented here. For the primary

\footnotetext{
$27 \mathrm{http}: / /$ cosmologist.info/cosmomc

$28 \mathrm{http}: / /$ pole.uchicago.edu/public/data/reichardt11/
}

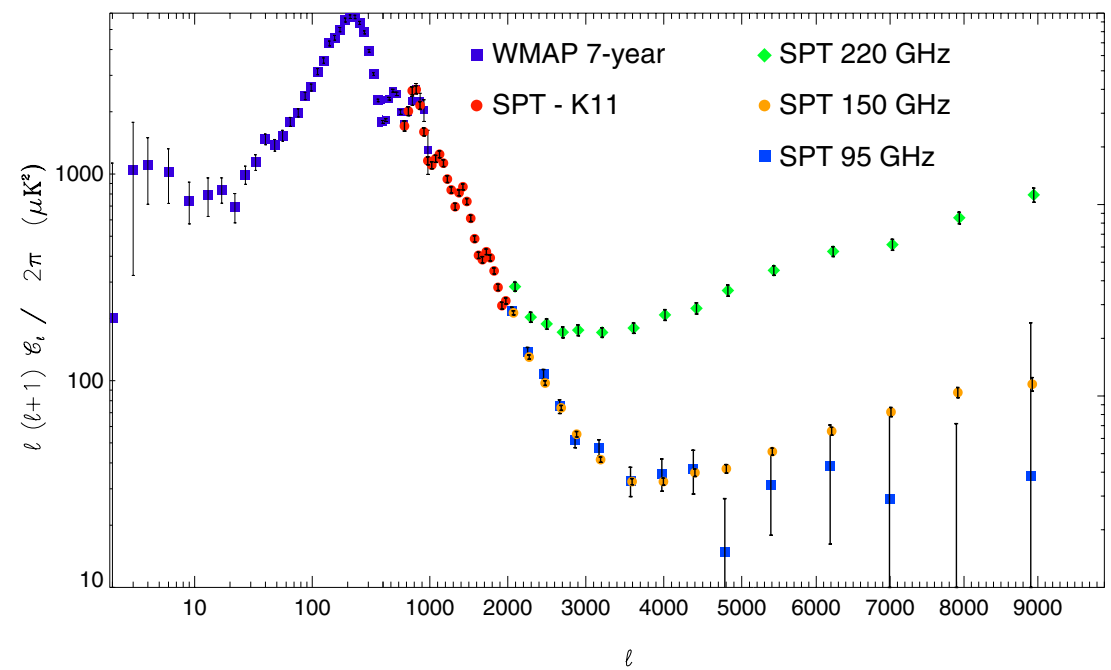

Figure 2. WMAP7 and SPT bandpowers. The SPT bandpowers at $\ell \leqslant 2000$ are taken from K11 and are at $150 \mathrm{GHz}$ only. At $\ell \geqslant 2000$, we show the bandpowers at 95,150 , and $220 \mathrm{GHz}$ measured with SPT in this work. Below $\ell=2000$, the primary CMB anisotropy is dominant at all frequencies. On smaller scales, the CIB, radio sources, and secondary CMB anisotropies contribute to the signal. With the SPT source masking, the CIB is the largest source of power on sub-arcminute scales at 150 and $220 \mathrm{GHz}$. Due to the relative spectral behavior of the CIB and synchrotron emission, the $95 \mathrm{GHz}$ bandpowers also have a significant contribution from radio sources.

(A color version of this figure is available in the online journal.) 


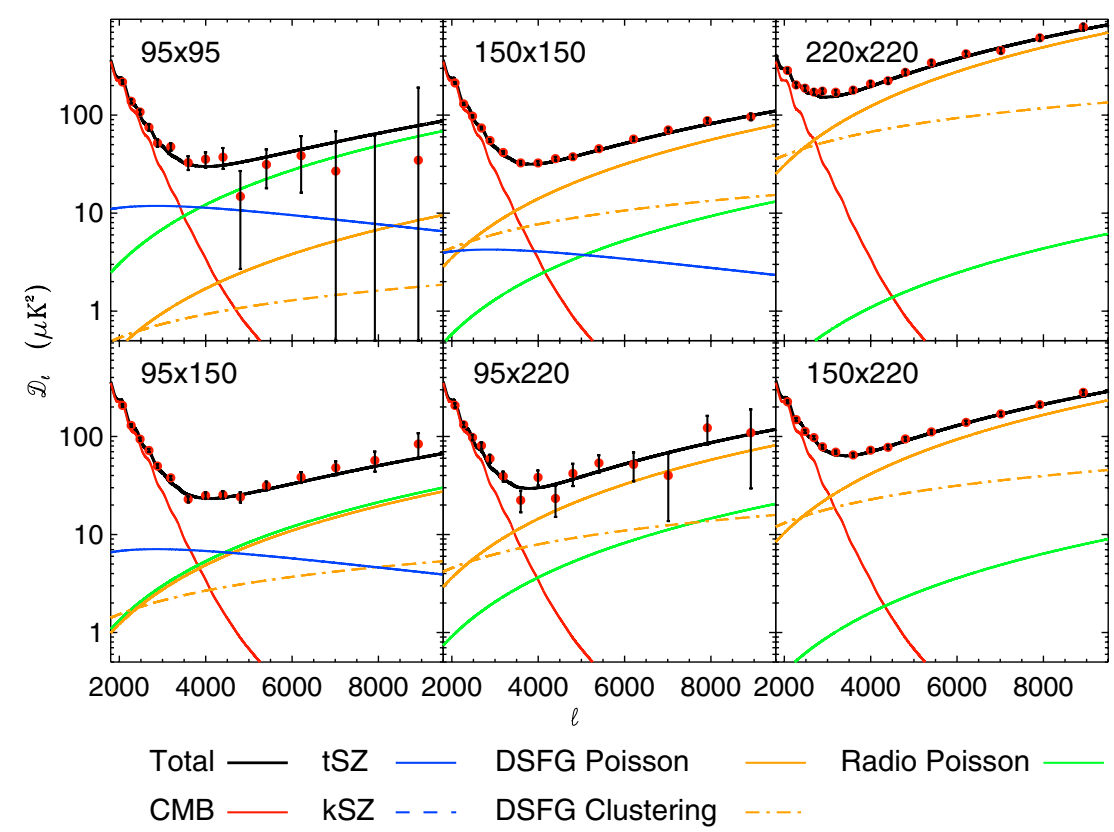

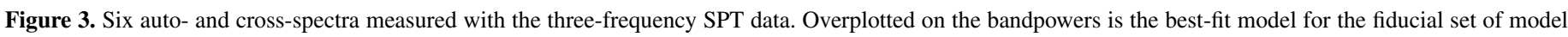

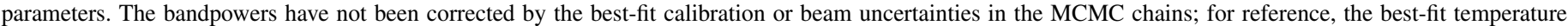

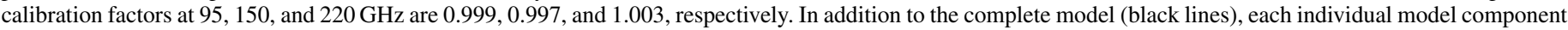

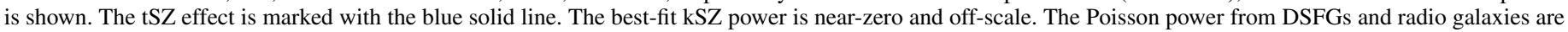
shown by solid orange and green lines, respectively. The clustered component to the DSFGs is shown with an orange dot-dashed line.

(A color version of this figure is available in the online journal.)

CMB, we include both WMAP7 (Larson et al. 2011) and the recent large-scale SPT power spectrum (K11). We note that the same TOD are used in this work and K11. Hence, we restrict the K11 data to non-overlapping multipoles $(\ell<2000)$. We also subtract a Poisson term from the K11 bandpowers to adjust for the different source masking thresholds between the two works (for details see Section 5.5). All parameter chains include low-redshift measurements of the Hubble constant $H_{0}$ using the Hubble Space Telescope (Riess et al. 2011) and the BAO feature using SDSS and 2dFGRS data (Percival et al. 2010). Finally, we include Planck/HFI CIB measurements at $217 \mathrm{GHz}$ and in some cases $353 \mathrm{GHz}$ (Planck Collaboration et al. 2011). When comparing to the CIB model, we subtract the estimated Poisson radio contribution from the Planck/HFI CIB bandpowers.

In some MCMC chains, we also compare the measured SZ power with external measurements of low-redshift structure. We use local galaxy cluster abundances as measured by Vikhlinin et al. (2009), which directly and tightly constrain $\sigma_{8}$ and are consistent with other structure probes (Rozo et al. 2010; Mantz et al. 2010; Vanderlinde et al. 2010; Sehgal et al. 2011). Vikhlinin et al. (2009) use the abundance of local $(0.025<z<0.22)$ clusters to infer $\sigma_{8}\left(\Omega_{m} / 0.25\right)^{0.47}=0.813 \pm 0.013 \pm 0.024$, where the second set of errors is an estimate of the systematic uncertainty due to the uncertainty in the masses of the clusters. This constraint will be referred to as the X-ray $\sigma_{8}$ prior.

\subsection{Primary Cosmic Microwave Background Anisotropy}

We use the standard, six-parameter, spatially flat, lensed $\Lambda \mathrm{CDM}$ cosmological model to predict the primary CMB temperature anisotropy. The six parameters are the baryon density $\Omega_{b} h^{2}$, the density of cold dark matter $\Omega_{c} h^{2}$, the optical depth to recombination $\tau$, the angular scale of the sound horizon at last scattering $\Theta_{s}$, the amplitude of the primordial density fluctuations $\ln \left[10^{10} A_{s}\right]$, and the scalar spectral index $n_{s}$.
We calculate the primary CMB power spectrum in the MCMC chains using PICO (Fendt \& Wandelt 2007a, 2007b) instead of CAMB (Lewis et al. 2000) for speed. With lensing on, we find chains run with PICO (and the fast WMAP likelihood code (Dvorkin \& Hu 2010) modified for the latest WMAP7 likelihood) to be roughly 2000 times faster than ones run with CAMB. PICO allows us to replace the unlensed spectra plus "lensing template" approximation used in L10 and S11 with the full lensing treatment. We have trained PICO using the 2011 January version of CAMB with lensing including nonlinear corrections turned on. Note that we observe no change to the foreground or secondary anisotropy constraints from the current data when using the former lensing template approximation. However, reducing the lensing power at $\ell=3000$ (by turning off lensing or neglecting nonlinear effects) would increase the inferred kSZ power by an approximately equal amount. For instance, neglecting nonlinear effects would increase the kSZ power by $\sim 1 \mu \mathrm{K}^{2}$.

\subsection{Thermal Sunyaev-Zel'dovich Anisotropy}

We adopt two different models for the tSZ power spectrum. S11 considered four tSZ models: a model by Sehgal et al. (2010) which predicts high levels of $\mathrm{tSZ}$ power and three models which all predicted lower tSZ powers (Battaglia et al. 2010; Shaw et al. 2010; Trac et al. 2011). Here, we have limited the model set to one from each group: the high power model by Sehgal et al. (2010) and the model by Shaw et al. (2010) from the lower set.

We use the tSZ power spectrum predicted by Shaw et al. (2010) as the baseline model. Shaw et al. (2010) investigate the impact of cluster astrophysics on the tSZ power spectrum using halo model calculations in combination with an analytic model for the ICM. These astrophysical effects - in particular, the inclusion of non-thermal pressure support and AGN feedbacktend to suppress the tSZ power (see also Battaglia et al. 2011b; 
Trac et al. 2011). We use the baseline model from that work (hereafter the Shaw model), which predicts $D_{3000}^{\mathrm{tSZ}}=4.3 \mu \mathrm{K}^{2}$ at $\ell=3000$ and $152.9 \mathrm{GHz}$. The assumed cosmological parameters are $\left(\Omega_{b}, \Omega_{m}, \Omega_{\Lambda}, h, n_{s}, \sigma_{8}\right)=(0.044,0.264,0.736$, $0.71,0.96,0.80)$. We use this model in all MCMC chains where another model is not explicitly specified.

We also consider the tSZ power spectrum model presented by Sehgal et al. (2010, hereafter the Sehgal model). Sehgal et al. (2010) combined the semi-analytic model for the intracluster medium (ICM) of Bode et al. (2009) with a cosmological $\mathrm{N}$-body simulation to produce simulated tSZ and kSZ maps from which the template power spectra were measured. The assumed cosmological parameters are the same as with the Shaw model. At $\ell=3000$, this model predicts $D_{3000}^{\mathrm{tSZ}}=7.4 \mu \mathrm{K}^{2}$ of tSZ power at $152.9 \mathrm{Ghz}$.

The principal difference between the two models is the inclusion by Shaw et al. (2010) of non-thermal pressure support from random gas motions. Bode et al. (2009) calibrated their intracluster gas model parameters by matching their model predictions to observed low-redshift X-ray scaling relations and radial profiles; Shaw et al. (2010) updated this analysis having included a radially dependent non-thermal pressure component (and its effect on hydrostatic mass estimates), calibrated from the results of hydrodynamical simulations (Lau et al. 2009). The result was a significantly suppressed thermal pressure fraction-particularly outside of the central regions of clusters-compared to the Bode et al. (2009) model, and thus a lower thermal SZ power spectrum amplitude than that produced by the Sehgal et al. (2010) simulations.

As described in S11, the model of Shaw et al. (2010) is also used to rescale both tSZ model templates as a function of cosmological parameters. Around the fiducial cosmological model, the tSZ amplitude roughly scales as

$$
D^{\mathrm{tSZ}} \propto\left(\frac{h}{0.71}\right)^{1.7}\left(\frac{\sigma_{8}}{0.80}\right)^{8.3}\left(\frac{\Omega_{b}}{0.044}\right)^{2.8} .
$$

Both tSZ models exhibit a similar angular scale dependence over the range of multipoles to which SPT is sensitive. When the normalization of each model is allowed to vary, we detect similar tSZ power for both (see Table 3). However, as we discuss in Section 7.2, the predicted model amplitude is crucial in interpreting the detected $\mathrm{tSZ}$ power as a constraint on $\sigma_{8}$ and also leads to significant changes in the fit quality when the tSZ power is fixed to cosmologically scaled model expectations.

\subsection{Kinetic Sunyaev-Zel'dovich Anisotropy}

Thomson scattering between CMB photons and clouds of free electrons that have a coherent bulk velocity produces fluctuations in the observed brightness temperature of the CMB. This effect, known as the kinetic Sunyaev-Zel'dovich (kSZ) effect, leads to hot or cold spots, depending on whether the ionized gas is moving toward or away from the observer. The shift is identical to a change in the CMB blackbody temperature, except for tiny relativistic corrections.

KSZ temperature anisotropy requires perturbations in the free electron density. This can be due to local perturbations in the baryon density, $\delta_{\mathrm{b}}=\rho_{\mathrm{b}} / \bar{\rho}_{\mathrm{b}}-1$ (where $\bar{\rho}_{b}$ is the mean baryon density in the universe), or ionization fraction, $\delta_{x}=x_{e} / \bar{x}_{e}-1$. Perturbations that are correlated with the large-scale velocity field will produce an observable change in temperature of the CMB blackbody. The total contribution to the temperature anisotropy is given by the integral over conformal time,

$$
\frac{\Delta T_{\mathrm{kSZ}}}{T_{\mathrm{CMB}}}(\hat{\mathbf{n}})=\sigma_{T} \bar{n}_{e, 0} \int d \eta\left[a^{-2} e^{-\tau(\eta)} \bar{x}_{e}(\eta)\right] \hat{\mathbf{n}} \cdot \mathbf{q},
$$

where $\sigma_{T}$ is Thomson scattering cross-section, $a$ is the scale factor, $\tau(\eta)$ is the optical depth from the observer to conformal time $\eta, \bar{n}_{e, 0}$ is the mean electron density of the universe at the present day, and $\hat{\mathbf{n}}$ is the line-of-sight unit vector. Finally,

$$
\mathbf{q}=\left(1+\delta_{x}\right)\left(1+\delta_{\mathrm{b}}\right) \mathbf{v},
$$

where $\mathbf{v}$ represents the peculiar velocity of free electrons at $\eta$.

The kSZ power spectrum can be broken down loosely into two components. The first is the kSZ signal from the postreionization epoch, $z<z_{\text {end }}$, where we define $z_{\text {end }}$ as the redshift at which hydrogen reionization is complete. The second component comes from the epoch of reionization itself. Models of inhomogeneous (or patchy) reionization predict bubbles of free electrons around UV-emitting sources embedded in an otherwise neutral medium. Any large-scale bulk velocity of these bubbles will impart a temperature anisotropy onto the $\mathrm{CMB}$. The $\mathrm{kSZ}$ signal from reionization is thus sensitive to fluctuations in both the gas density and ionization fraction. Note that in the case of instantaneous reionization, the postreionization signal is the only source of $\mathrm{kSZ}$ power.

We henceforth refer to the post-reionization component as the "homogeneous kSZ" signal and that from the reionization epoch as the "patchy kSZ" signal. We now discuss our modeling of each in more detail.

\subsubsection{Homogeneous Kinetic Sunyaev-Zel'dovich Anisotropy}

For the homogeneous (or post-reionization) kSZ signal, we adopt the non-radiative (NR) and cooling plus star formation (CSF) models presented by Shaw et al. (2011). These models are constructed by calibrating an analytic model for the homogeneous kSZ power spectrum with two hydrodynamical simulations. The first simulation was run in the non-radiative regime, while the second included metallicity-dependent radiative cooling and star formation. Shaw et al. (2011) measured the power spectrum of gas density fluctuations in both simulations over a range of redshifts and used this to calculate the $\mathrm{kSZ}$ power spectrum for each case. They argue that these two cases are likely to bracket the true homogeneous $\mathrm{kSZ}$ power. For our fiducial cosmology (detailed in Section 5.2) at $\ell=3000$, the NR template predicts $2.4 \mu \mathrm{K}^{2}$, whereas the CSF simulation predicts $1.6 \mu \mathrm{K}^{2}$. The scaling of these models with cosmological parameters is given approximately by

$$
\begin{aligned}
D^{\mathrm{kSZ}} \propto & \left(\frac{h}{0.71}\right)^{1.7}\left(\frac{\sigma_{8}}{0.80}\right)^{4.7}\left(\frac{\Omega_{b}}{0.044}\right)^{2.1} \\
& \times\left(\frac{\Omega_{m}}{0.264}\right)^{-0.4}\left(\frac{n_{s}}{0.96}\right)^{-0.2} .
\end{aligned}
$$

Note that, for both models, we assume that reionization occurs instantaneously at $z_{\text {end }}=8$. Both models also assume that helium remains neutral; if helium is singly ionized at the same time as hydrogen, the predicted power would increase by $16 \%$.

\subsubsection{Kinetic Sunyaev-Zel'dovich Anisotropy from Patchy Reionization Scenarios}

For the patchy kSZ signal, we adopt the model presented by Z11 and briefly summarized here. This model starts from 
a matter simulation. For every position, the linear matter overdensity is calculated within some radius. The halo collapse fraction is estimated from this overdensity and translated into an expected number of ionizing photons based on the number of collapsed halos above the atomic cooling threshold mass. The number of ionizing photons is then compared to the number of hydrogen atoms within this sphere. If there are sufficient ionizing photons, the sphere is labeled ionized. If not, a smaller radius is set and the algorithm repeated until the resolution of the simulation box is reached. The resulting ionization field is used in conjunction with the underlying density and velocity fields to compute the patchy kSZ power spectrum.

As discussed in Z11, the detailed reionization history used for the kSZ template is unimportant, since the template shape is robust to the duration and the mean redshift of reionization. The amplitude of the patchy kSZ signal is nearly proportional to the duration of reionization (with a mild redshift dependence), making it an excellent probe of reionization. The kSZ effect is complementary to the large-scale CMB polarization anisotropy (the reionization bump) that provides a constraint on the global timing of reionization, and to QSO and Ly $\alpha$ emitter observations that study the very end of reionization. For the fiducial model template provided by $\mathrm{Z} 11$ and used in this work, reionization begins at $z=11$ and concludes at $z=8$.

\subsection{Cosmic Infrared Background}

The CIB is produced by thermal emission from DSFGs over a very broad range in redshift (Lagache et al. 2005; Marsden et al. 2009). The dust grains, ranging in size from a few molecules to $0.1 \mathrm{~mm}$, absorb light at wavelengths smaller than their size, and re-radiate it at longer wavelengths. The result of this absorption is roughly equal amounts of energy in the CIB as in the unprocessed starlight that makes up the optical/UV background (Dwek \& Arendt 1998; Fixsen et al. 1998).

The frequency dependence of the CIB (and radio galaxies in Section 5.5) is naturally discussed and modeled in units of flux density (Jy) rather than CMB temperature units. However, as the power spectrum is calibrated in CMB temperature units, we need to determine the ratio of powers between different frequency bands in CMB temperature units. To calculate the ratio of power in the $v_{i} \times v_{j}$ cross-spectrum to the power at (the arbitrary frequency) $\nu_{0}$ in units of CMB temperature squared, we multiply the ratio of flux densities by

$$
\epsilon_{\nu_{1}, \nu_{2}} \equiv \frac{\left.\left.\frac{d B}{d T}\right|_{\nu_{0}} \frac{d B}{d T}\right|_{\nu_{0}}}{\left.\left.\frac{d B}{d T}\right|_{\nu_{i}} \frac{d B}{d T}\right|_{\nu_{j}}}
$$

where $B$ is the CMB blackbody specific intensity evaluated at $T_{\mathrm{CMB}}$, and $v_{i}$ and $v_{j}$ are the effective frequencies of the SPT bands. Note that $v_{i}$ may equal $v_{j}$. The effective frequencies of the SPT bandpasses for each foreground are presented in Section 5.7 .

\subsubsection{Poisson Term}

Statistical fluctuations in the number of DSFGs lead to a Poisson distribution on the sky. DSFGs are effectively point sources at these angular scales, so they contribute a constant $C_{\ell}$ (thus $D_{\ell} \propto \ell^{2}$ ) to the SPT bandpowers. The bulk of the millimeter-wavelength CIB anisotropy power is expected to come from faint ( $\lesssim 1 \mathrm{mJy}$ ) DSFGs - so the residual CIB power is largely independent of the source mask flux threshold. This is a key assumption in combining the Planck/HFI and SPT data as the flux cuts differ by a factor of eight at $220 \mathrm{GHz}$. The $217 \mathrm{GHz}$ Planck/HFI cut is at $160 \mathrm{mJy}$ while the $150 \mathrm{GHz}$ SPT flux cut of $6.4 \mathrm{mJy}$ scales to approximately $22 \mathrm{mJy}$ at $220 \mathrm{GHz}$. We test the impact of the flux cut with the modeled source counts of Béthermin et al. (2011) and Negrello et al. (2007), and find the two models predict a difference in the Poisson power of $4 \%$ or $16 \%$, respectively. Hence in the model fitting, we scale the modeled DSFG Poisson power up by $10 \%$ when comparing the model to the Planck/HFI CIB bandpowers. We have tested changing this assumption by tens of percent and find the cosmological constraints are insensitive to this-the Poisson term is subdominant at all multipoles $(\ell \in[80,2240])$ in the Planck/HFI bandpowers.

The Poisson power spectrum of the DSFGs can be written as

$$
D_{\ell, v_{1}, \nu_{2}}^{p}=D_{3000}^{p} \epsilon_{\nu_{1}, \nu_{2}} \frac{\eta_{\nu_{1}} \eta_{\nu_{2}}}{\eta_{\nu_{0}} \eta_{\nu_{0}}}\left(\frac{\ell}{3000}\right)^{2}
$$

where $\eta_{v}$ encodes how the CIB brightness scales with observing frequency (see Section 5.4.3). $D_{3000}^{p}$ is the amplitude of the Poisson DSFG power spectrum at $\ell=3000$ and frequency $v_{0}$. Note that we neglect the decohering effect of frequency dependence varying from source to source. Hall et al. (2010) argued that the spectral index variation in the DSFG population was less than $\sigma_{\alpha}=0.7$. This level of variation has a negligible effect on the inferred Poisson powers across the SPT frequency bands.

\subsubsection{Clustered Term}

DSFGs (and galaxies in general) trace the mass distribution, so they are spatially correlated. This leads to a "clustering" term in the DSFG power spectrum in addition to the Poisson term. On the physical scales of relevance, galaxy correlation functions are surprisingly well approximated by a power law. The clustering shape we consider is thus a phenomenologically motivated power law with index chosen to match the correlation properties observed for Lyman-break galaxies at $z \sim 3$ (Giavalisco et al. 1998; Scott \& White 1999). This form has been used previously by a number of millimeter- and submillimeter-wavelength experiments (Viero et al. 2009; Dunkley et al. 2011; Planck Collaboration et al. 2011; Addison et al. 2012; S11). We adopt this "power-law" model to represent the nonlinear regime and use a clustering template of the form $D_{\ell}^{c} \propto \ell^{0.8}$ in all cases unless specified.

In Section 9.1, we consider two alternate forms for the clustering term. First, we free the power-law exponent, i.e., $D_{\ell}^{c} \propto \ell^{\gamma}$ with $\gamma$ free. Second, we add the linear-theory model by Hall et al. (2010) with a free amplitude to the power-law clustering term. The fiducial model in that work was calculated assuming that light is a biased tracer of mass fluctuations calculated in linear perturbation theory, with redshift and scaleindependent bias. Further, the model assumed all galaxies have the same graybody SED, and the redshift distribution of the luminosity density was given by a parameterized form, with parameters adjusted to fit SPT, BLAST, and Spitzer power spectrum measurements. We find that the angular template shape depends only slightly on the observing frequency and choose to use the $150 \mathrm{GHz}$ model spectrum. Here we refer to this power spectrum shape as the "linear theory" template. As will be discussed in Section 9, the data strongly prefer the power-law form over the linear theory clustering template. The data are also consistent with the assumed power-law exponent of $\gamma=0.8$. 


\subsubsection{Frequency Dependence}

Unless otherwise noted, all results presented in this work assume the modified blackbody (modified BB) model for the frequency dependence of the CIB:

$$
\eta_{v}=v^{\beta} B_{v}(T)
$$

where $B_{v}(T)$ is the blackbody spectrum for temperature $T$, and $\beta$ is an effective dust emissivity index. $T$ and $\beta$ are free parameters with uniform priors $T \in[5,35 \mathrm{~K}]$ and $\beta \in[0,2]$. The upper limit on the temperature prior is unimportant because the SPT frequencies are in the Rayleigh-Jeans tail of $>15 \mathrm{~K}$ objects. Therefore, there would be a perfect degeneracy between $30 \mathrm{~K}$ and $100 \mathrm{~K}$ dust. We have tested instead fixing the temperature to 9.7 K with a wider $\beta$ range as was done in Addison et al. (2012) and found little effect on the results reported in this work.

For some results, we also examine the single-SED model presented by Hall et al. (2010) (hereafter the single-SED model); however, we do not find substantive differences in the results from the modified BB model. This model assumes a single SED for all DSFGs. This SED is a modified blackbody with $T=$ $35 \mathrm{~K}$ and $\beta=2$. The DSFGs have a density as a function of redshift parameterized by a Gaussian with mean $z_{\mathrm{c}}$ and variance $\sigma_{z}^{2}$. The number counts are set to zero at $z<0$. We fix all but one parameter in the model; we allow $z_{\mathrm{c}}$ to vary freely. As in Hall et al. (2010), we set $\sigma_{z}=2$. The frequency dependence predicted by the single-SED model has a slight $\ell$-dependence; we ignore this and use the frequency scaling at $\ell=3000$. The single-SED model has one fewer parameter and is more easily interpretable physically than the modified BB model. However, the data are in mild tension with the model - the preferred $z_{\mathrm{c}}$ is near the prior cutoff at 0 . This does not degrade the quality of the fit to the data, but it leads to a one-sided distribution of CIB spectral indices, which results in a slight artificial reduction in some parameter uncertainties.

A simplifying assumption we make for the analyses presented here is that the various components of the CIB (Poisson and clustering terms) have the same frequency dependence. Even in the context of the single-SED model, we expect their spectral indices to differ to some degree, because the weighting of sources as a function of redshift will be different for the two terms. Our assumption here is, effectively, that the redshift distributions of the source populations giving rise to the Poisson and clustered components are not sufficiently different as to cause a significant difference in frequency dependence. We have tested allowing separate frequency dependencies for the Poisson and clustered CIB terms and find minimal impact on the SZ constraints.

We have also checked whether the higher frequency, 217 and $353 \mathrm{GHz}$, Planck/HFI CIB bandpowers (Planck Collaboration et al. 2011) are consistent with the assumption of a single frequency dependence for the Poisson and clustered terms. If the frequency dependence of each term was different, then we would expect to see variations in the CIB power ratio between $217 \mathrm{GHz}$ and $353 \mathrm{GHz}$ as a function of angular scale since the relative power in each term is $\ell$-dependent. We find that the CIB power ratio is consistent with a single spectral index with $\alpha_{217 / 353}=3.22 \pm 0.08$. With (9-1) degrees of freedom (dof), the null hypothesis of a single spectral index has $\chi^{2}=8.0$ - the probability to exceed this $\chi^{2}$ is 0.44 . This spectral index is flatter than preferred by the lower frequency SPT data; however, we expect the CIB frequency dependence to flatten toward higher frequencies as the rest-frame emission frequency approaches the peak of the graybody dust emission.

\subsection{4. tSZ-CIB Correlations}

As tracers of the same dark matter distribution, we expect some degree of correlation between the tSZ and CIB power spectra. In previous papers, we have argued that the correlation is likely to be small since most of the DSFGs contributing to the CIB are in the field rather than in the massive galaxy clusters that contribute most of the tSZ power. Unfortunately, observational constraints on the IR flux (and therefore tSZ-CIB correlation) are largely limited to low-redshift, high-mass clusters. The observations are also at frequencies close to the peak of the CIB emission, introducing a significant scaling uncertainty in translating the results to the longer wavelengths important for tSZ surveys. The simulations of Sehgal et al. (2010) show that a model with significant correlations at $148 \mathrm{GHz}$ can be constructed without violating current observational constraints by concentrating the CIB galaxies in the lower-mass, higherredshift galaxy clusters.

We adopt a simplified treatment of tSZ-CIB correlations in this work. We assume that the correlation, $\xi$, is independent of angular multipole, and that both the Poisson and clustering CIB components correlate with tSZ fluctuations. Note that the choice of which CIB terms correlate does not significantly change the resulting $\mathrm{tSZ}$ and $\mathrm{kSZ}$ constraints, although it does rescale the amplitude of the inferred correlation.

The tSZ-CIB correlation introduces a term to the model of the form

$$
\left.\left.D_{\ell, v_{1}, \nu_{2}}^{\mathrm{tSZ}-\mathrm{CIB}}=\xi\left(\sqrt{(} D_{\ell, v_{1}, v_{1}}^{\mathrm{tSZ}} D_{\ell, v_{2}, \nu_{2}}^{\mathrm{CIB}}\right)+\sqrt{(} D_{\ell, v_{2}, \nu_{2}}^{\mathrm{tSZ}} D_{\ell, v_{1}, \nu_{1}}^{\mathrm{CIB}}\right)\right)
$$

Here $D_{\ell, v_{1}, v_{2}}^{\mathrm{tSZ}-\mathrm{CIB}}$ is the power due to correlations (which should be negative below the tSZ null of $217 \mathrm{GHz}$, i.e., $\xi<0$ ), $D_{\ell, v_{1}, v_{1}}^{\mathrm{tSZ}}$ is the tSZ power spectrum, and $D_{\ell, v_{1}, v_{1}}^{\mathrm{CIB}}$ is the sum of the Poisson and clustered CIB components.

\subsection{Radio Galaxies}

High-flux point sources in the SPT maps are coincident with known radio sources from long-wavelength catalogs (e.g., SUMSS; Mauch et al. 2003) and have spectral indices consistent with synchrotron emission (Vieira et al. 2010). The Poisson radio source term can be expressed as

$$
D_{\ell, \nu_{1}, \nu_{2}}^{r}=D_{3000}^{r} \epsilon_{\nu_{1}, \nu_{2}} \eta_{\nu_{1}, \nu_{2}}^{\alpha_{r}+0.5 \ln \left(\eta_{\nu_{1}, \nu_{2}}\right) \sigma_{r}^{2}}\left(\frac{\ell}{3000}\right)^{2}
$$

where $\eta_{v_{1}, v_{2}}=\left(v_{1} v_{2} / v_{0}^{2}\right)$ is the ratio of the frequencies of the spectrum to the base frequency. $D_{3000}^{r}$ is the amplitude of the Poisson radio source power spectrum at $\ell=3000$ and frequency $v_{0}$. The mean spectral index of the radio source population is $\alpha_{r}$ with scatter $\sigma_{r}$.

The radio source power $\left(D_{3000}^{r}\right)$ in the map depends approximately linearly on the flux above which discrete sources are masked. This reflects the fact that $S^{2} d N / d S$ for synchrotron sources is nearly independent of $S$ (e.g., de Zotti et al. 2005). As a result, the Poisson radio source power in the K11 bandpowers (which had a higher flux cutoff) is expected to be $9.2 \pm 2.4 \mu \mathrm{K}^{2}$ higher than in the $150 \mathrm{GHz}$ bandpowers presented here. We correct for this by subtracting a Poisson term with amplitude $D_{3000}=9.2 \mu \mathrm{K}^{2}$ from the $\mathrm{K} 11$ bandpowers in the parameter fits. Note that the restriction to $\ell<2000$ that we impose on the 
K11 bandpowers renders the uncertainty on the Poisson power insignificant. We have tested varying the subtracted Poisson power and found no impact on the results.

Following the treatment in S11, we apply a strong prior to the Poisson radio source term due to sources below the SPT detection threshold. First, we fix the mean spectral index $\left(\alpha_{r}=-0.53\right)$ and scatter $\left(\sigma_{r}=0\right)$. Second, based on the de Zotti et al. (2005) source count model, we apply a Gaussian prior to the $150 \mathrm{GHz}$ amplitude of $1.28 \pm 0.19 \mu \mathrm{K}^{2}$. We have tested the impact of removing the radio priors. The data prefer parameter values consistent with the priors, and there are no significant changes to the CIB and SZ constraints. The strong radio priors are included in all cases presented here.

As discussed in Hall et al. (2010), the clustering of radio galaxies at SPT frequencies is likely to be negligible. Clustering on scales of a few arcminutes is a $\sim 5 \%$ modulation of the mean, and the intensity of the radio source mean is quite low. Furthermore in the unified model of active galactic nuclei, the dominant sources at $>90 \mathrm{GHz}$ (blazars and flat spectrum radio quasars) are active galactic nuclei with their jets aligned toward the observer. The effect of random alignment would serve to further dilute the clustering signal. Supporting this argument is conservative extrapolations of upper limits on arcminute scale power at $30 \mathrm{GHz}$ (Sharp et al. 2010). If entirely due to radio point-source power, the $30 \mathrm{GHz}$ limit translates to an upper limit of $0.1 \mu \mathrm{K}^{2}$ at $150 \mathrm{GHz}$. We do not include a clustered radio component in any results in this work.

\subsection{Galactic Cirrus}

Galactic cirrus is the final (and smallest) foreground we include in our modeling. Following the cirrus treatment in Hall et al. (2010) and K11, we cross-correlate the SPT maps with the model 8 galactic dust predictions of Finkbeiner et al. (1999). Galactic cirrus is detected in all frequency bands at a significance of $\sim 3 \sigma$ with an angular dependence of

$$
D_{\ell, \nu_{1}, \nu_{2}}^{\mathrm{cir}}=D_{3000}^{\mathrm{cir}, \nu_{1}, \nu_{2}}\left(\frac{\ell}{3000}\right)^{-1.2}
$$

The measured powers are $0.16,0.21$, and $2.19 \mu \mathrm{K}^{2}$ at 95,150 , and $220 \mathrm{GHz}$. Note that the $150 \mathrm{GHz}$ amplitude is different from the value quoted in K11 because of the different field weights in the two analyses. We fix the amplitude of the galactic cirrus in each frequency band; allowing it to vary with a prior based on the measurement uncertainty has no impact on any parameter constraints.

\subsection{Effective Frequencies of the SPT Bands}

Throughout this work, we refer to the SPT frequencies as 95, 150, and $220 \mathrm{GHz}$. The data are calibrated to CMB temperature units, hence the effective frequency is irrelevant for a CMB-like source. However, the effective band center will depend (weakly) on the source spectrum for other sources. The actual SPT spectral bandpasses are measured by Fourier transform spectroscopy, and the effective band center frequency is calculated for each source, assuming a beam-filling source with a nominal frequency dependence. The bandpasses differ slightly from 2008 to 2009 due to the focal plane refurbishment. We calculate the effective frequency for each year's spectral bandpass and average them according to the yearly weights presented in Section 2.1. For an $\alpha=-0.5$ (radio-like) source spectrum, we find band centers of 95.3, 150.2, and $214.1 \mathrm{GHz}$.
Table 2

Delta $\chi^{2}$ for Model Components

\begin{tabular}{lrc}
\hline \hline Term & dof & $\Delta \chi^{2}$ \\
\hline CMB & 6 & (reference) \\
DSFG Poisson & 3 & -1645 \\
DSFGs NL clustering & 1 & -218 \\
Radio Poisson & 0 & -164 \\
tSZ & 1 & -55 \\
kSZ & 1 & 0 \\
\hline Single SED & -1 & 0 \\
$\alpha_{p} \neq \alpha_{c}$ & 2 & -4 \\
tSZ-CIB correlation & 1 & -2 \\
$\ell^{\gamma}$ clustered DSFGs & 1 & 0 \\
LT clustered DSFGs & 1 & 0 \\
\hline
\end{tabular}

Notes. Improvement to the best-fit $\chi^{2}$ as additional terms are added to the model. Terms above the double line are included in the baseline model, with each row showing the improvement in likelihood relative to the row above it. For rows below the double line, the $\Delta \chi^{2}$ is shown relative to the baseline model rather than the row above it. Here we have assumed the modified BB frequency scaling of the CIB except for the row labeled "single SED," which assumes the alternate single-SED frequency scaling. The row labeled " $\alpha_{p} \neq \alpha_{c}$ " allows the Poisson and clustered power from DSFGs to have independent frequency scalings. The row labeled "tSZ-CIB correlation" introduces a correlation coefficient between the tSZ and CIB power spectra. The last two rows add freedom to the clustered DSFG model shape, either by varying the power-law exponent (" $\ell \gamma$ clustered DSFGs") or adding a free amplitude linear theory template ("LT clustered DSFGs").

For an $\alpha=3.5$ (dust-like) source spectrum, we find band centers of $97.9,153.8$, and $219.6 \mathrm{GHz}$. The band centers in both cases drop by $\sim 0.2 \mathrm{GHz}$ if we reduce $\alpha$ by 0.5 . We use these radiolike and dust-like band centers to calculate the frequency scaling between the SPT bands for the radio and DSFG terms. For a tSZ spectrum, we find band centers of 97.6, 152.9, and $218.1 \mathrm{GHz}$. The ratio of tSZ power in the $95 \mathrm{GHz}$ band to that in the $150 \mathrm{GHz}$ band is 2.78 . The $220 \mathrm{GHz}$ band center is effectively at the tSZ null; the ratio of tSZ power in the $220 \mathrm{GHz}$ band to that in the $150 \mathrm{GHz}$ band is 0.00004 for a non-relativistic tSZ spectrum.

\section{RESULTS}

\subsection{Baseline Model Results}

We adopt a baseline model with the six $\Lambda$ CDM parameters and six additional parameters for the secondary CMB anisotropies and foregrounds described in Section 5. Four of these extra parameters represent the power in the tSZ effect, kSZ effect, DSFG Poisson term, and DSFG clustering term. The final two parameters describe the frequency dependence of the DSFG anisotropy. All DSFG terms are assumed to have the same frequency scaling, based on the modified BB model. As templates for the angular shape of $\mathrm{tSZ}$ and $\mathrm{kSZ}$ power spectra, we use the Shaw et al. (2010) tSZ model and Shaw et al. (2011) CSF kSZ model. The DSFG Poisson power (like all unresolved Poisson power) has the shape $D_{\ell} \propto \ell^{2}$, while the DSFG clustering term is described by the power-law $D_{\ell} \propto \ell^{0.8}$ template presented in Section 5.4.2. Figure 3 plots the best-fit values for each component on top of the SPT bandpowers.

Table 2 shows the improvement in the quality of the fits with the sequential introduction of free parameters to the original $\Lambda \mathrm{CDM}$ primary $\mathrm{CMB}$ model. Adding additional free parameters 
Table 3

SZ Constraints

\begin{tabular}{lcccc}
\hline \hline tSZ Model & kSZ Model & CIB Model & $\begin{array}{c}D_{3000}^{\mathrm{tSZ}} \\
\left(\mu \mathrm{K}^{2}\right)\end{array}$ & $\begin{array}{c}D_{3000}^{\mathrm{kSZ}} \\
\left(\mu \mathrm{K}^{2}\right)\end{array}$ \\
\hline Shaw & CSF & Modified BB & $3.65 \pm 0.69$ & $<2.8$ \\
Shaw & CSF & Modified BB w. correlations & $3.26 \pm 1.06$ & $<6.7$ \\
Shaw & Patchy & Modified BB w. correlations & $3.66 \pm 0.86$ & $<5.7$ \\
Shaw & Fixed CSF & Modified BB & $3.34 \pm 0.58$ & $(1.57)$ \\
\hline
\end{tabular}

Notes. Measured tSZ power and 95\% confidence upper limits on the kSZ power at $\ell=3000$ in the SPT $150 \mathrm{GHz}$ band. Two tSZ model templates (the Shaw and Sehgal models) and two kSZ models (the CSF homogeneous kSZ model and the patchy reionization $\mathrm{kSZ}$ model) have been considered. In most cases, we show results only for the Shaw tSZ template and CSF kSZ template as the other cases are essentially identical-indicating the data are insensitive to the differences in the modeled angular dependencies. In all cases, the modified BB model is assumed for the CIB frequency scaling. As an extension to the CIB model, we present constraints when correlations are allowed between the tSZ and CIB. This correlation allows twice the kSZ power and weakens the tSZ power constraint by 50\%. The results depend somewhat on the $\mathrm{kSZ}$ template when tSZ-CIB correlations are introduced. The real kSZ spectrum should be a mixture of the two kSZ templates. Finally, we show the tSZ constraint derived when fixing the kSZ power to that predicted by the CSF homogeneous kSZ model. The CSF kSZ model predicts $1.57 \mu \mathrm{K}^{2}$ for the WMAP7 cosmology detailed in Section 5.3.1 and is scaled with cosmology according to the prescription in that section.

beyond the tSZ amplitude does not significantly improve the quality of the fits. However, small improvements are seen with the introduction of tSZ-CIB correlations or treating the clustered and Poisson frequency scalings as independent.

The additional parameters discussed in Section 5 are either fixed or constrained by strong priors. A Poisson population of radio galaxies is included with a fixed spectral index $\alpha_{r}=$ -0.53 , zero intrinsic scatter, and an amplitude of $D_{3000}^{r}=$ $1.28 \pm 0.19 \mu \mathrm{K}^{2}$. Weakening these radio priors-removing the amplitude prior and setting a uniform prior on the radio spectral index of $\alpha_{r} \in[-1.5,0]$ - does not change the inferred SZ levels. We always include the constant Galactic cirrus power described in Section 5.6. We assume no correlation between the tSZ effect and CIB in the baseline model. This assumption is relaxed in Section 6.2.1. The $\chi^{2}$ for the 90 SPT bandpowers (84 dof since the $\Lambda$ CDM parameters are essentially fixed by external data and there are six model parameters beyond $\Lambda \mathrm{CDM}$ ) is 73.3 ; the PTE for this $\chi^{2}$ is $79 \%$. We have tested fixing the $\Lambda$ CDM parameters to the best-fit values and find the derived constraints on the six extended model parameters are unchanged. This baseline model fits the SPT data well and provides the most concise interpretation of the data.

SZ parameter constraints for the baseline model and extensions are listed in Table 3 and are presented in Figure 4. For the tSZ power, we find $D_{3000}^{\mathrm{tSZ}}=3.65 \pm 0.69 \mu \mathrm{K}^{2}$, which is consistent with the predictions of the Shaw model. We find a strong $95 \%$ confidence level (CL) upperlimit on $\mathrm{kSZ}$ power at $D_{3000}^{\mathrm{kSZ}}<2.8 \mu \mathrm{K}^{2}$, although we stress that this limit is dependent on our DSFG modeling assumptions. We have applied a positivity prior to the $\mathrm{kSZ}$ power; without this prior, the median $\mathrm{kSZ}$ power is negative by $1.2 \sigma$. The SZ constraints are independent of the assumed template shape; we find similar power constraints for all tSZ and kSZ model templates considered.

The current tSZ and kSZ constraints are consistent with earlier measurements of the SZ power. S11 measured $D_{3000}^{\mathrm{tSZ}}+$ $0.5 D_{3000}^{\mathrm{kSZ}}=4.5 \pm 1.0 \mu \mathrm{K}^{2}$. The equivalent constraint from these data is (1.034) $D_{3000}^{\mathrm{tSZ}}+0.5 D_{3000}^{\mathrm{kSZ}}=4.27 \pm 0.58 \mu \mathrm{K}^{2}$. The multiplicative factor in parentheses before the tSZ term corrects for the different effective bandcenters in the two data sets. Dunkley et al. (2011) measured $D_{3000}^{\mathrm{tSZ}}+D_{3000}^{\mathrm{kSZ}}=6.8 \pm 2.9 \mu \mathrm{K}^{2}$, while these data prefer (1.17) $D_{3000}^{\mathrm{tSZ}}+D_{3000}^{\mathrm{kSZ}}=5.27 \pm 0.76 \mu \mathrm{K}^{2}$.
We note that the two analyses differ in that Dunkley et al. (2011) fixed the ratio of thermal and kinetic SZ power, while this ratio is free in this work. The three data sets show excellent consistency.

We detect both the DSFG Poisson and clustering power with high significance, with powers of $D_{3000}^{p}=7.54 \pm 0.38 \mu \mathrm{K}^{2}$ and $D_{3000}^{c}=6.25 \pm 0.52 \mu \mathrm{K}^{2}$, respectively, at $150 \mathrm{GHz}$. The effective spectral index from 150 to $220 \mathrm{GHz}$ of the DSFG power is found to be $\alpha=3.56 \pm 0.07$. The DSFG constraints are discussed in more detail in Section 9, and are in line with both theoretical expectations and previous work (H10; S11; Dunkley et al. 2011).

\subsection{Results for Alternative Models}

Here, we explore modifications to the baseline model and the impact on SZ and CIB constraints. First, we consider allowing a correlation between the tSZ effect and CIB, and show the impact on the derived SZ power. The SZ interpretation is robust to the other CIB model variants we examined. Second, we examine model extensions to the $\Lambda \mathrm{CDM}$ cosmology, and show that they do not affect the SZ and CIB parameters.

\subsection{1. tSZ-CIB Correlations}

We first consider the effect of a correlation between the tSZ and CIB as prescribed by Equation (17). Note that DSFG overdensities in galaxy clusters would lead to an anti-correlation since the tSZ effect causes a flux decrement at the SPT frequencies. The correlated power is highly degenerate with the $\mathrm{kSZ}$ and tSZ power as shown in Figure 5. We see some dependence on the kSZ template shape (not shown); the patchy template, which is falling instead of rising slightly across the angular multipoles of interest, is less degenerate with the correlation coefficient than the CSF homogeneous kSZ model. Increasing anti-correlation increases the allowed $\mathrm{kSZ}$ power, while reducing the $\mathrm{tSZ}$ power, until $\xi \sim-0.4$. The slope of the tradeoff between $\xi$ and $\mathrm{kSZ}$ power approaches zero for very negative values of $\xi$. This is because the correlated power is proportional to the tSZ power, and the tSZ power approaches zero in this limit. 


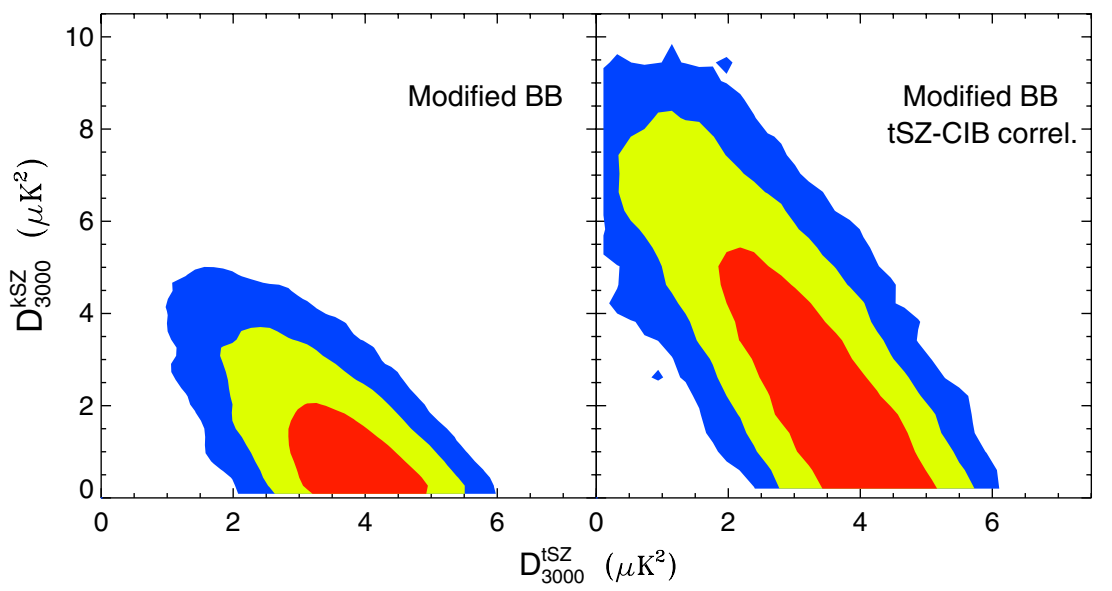

Figure 4. Two-dimensional likelihood surface for the tSZ and kSZ power in the SPT $150 \mathrm{GHz}$ band at $\ell=3000.1 \sigma, 2 \sigma$, and $3 \sigma$ constraints are shown in red, yellow, and blue respectively. The left panel shows the constraints for the modified BB CIB frequency scaling. The right panel displays the likelihood surfaces for the modified BB CIB frequency scaling with the introduction of free parameter, $\xi$, describing the correlations between the tSZ and CIB power spectra. The tSZ-CIB correlation is degenerate with the $\mathrm{kSZ}$ effect, thereby weakening the kSZ limits.

(A color version of this figure is available in the online journal.)

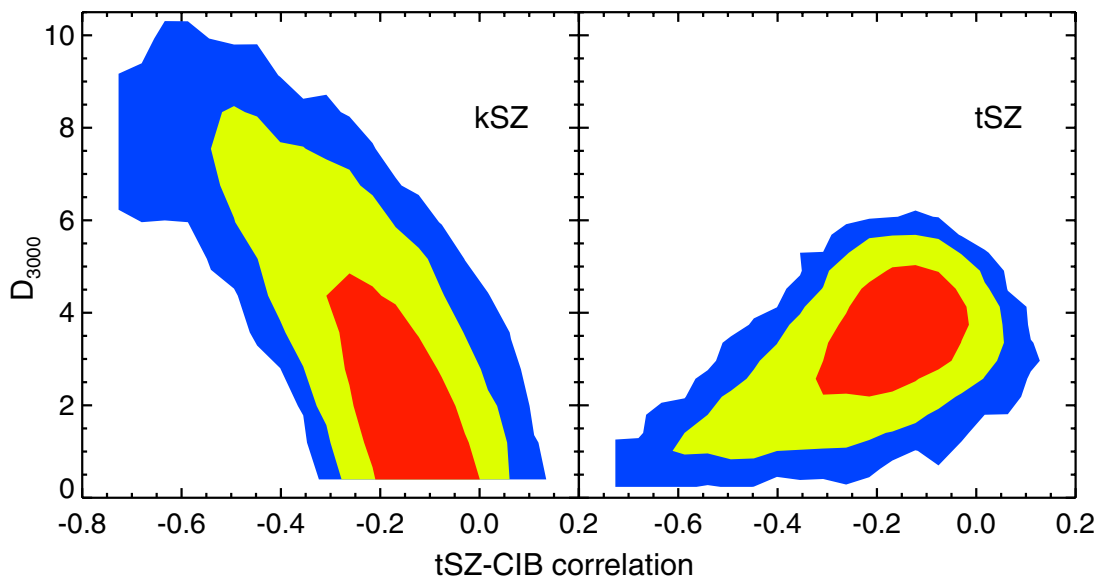

Figure 5. Two-dimensional likelihood curves for the correlation between the tSZ and CIB spectra vs. the kSZ power (left panel) or tSZ power (right panel) in $\mu \mathrm{K}^{2}$. The filled contours show the $1 \sigma, 2 \sigma$, and $3 \sigma$ constraints. The kSZ template consists of the sum of CSF homogeneous kSZ model and patchy kSZ contribution for a model where reionization begins at $z=11$ and ends at $z=8$. Increasing anti-correlation increases the allowed kSZ power and reduces the tSZ power. The data prefer anti-correlation suggesting that DSFGs are overdense in galaxy clusters.

(A color version of this figure is available in the online journal.)

Decreasing tSZ power with increasing anti-correlation contradicts our naive expectations. In single-frequency bandpowers (e.g., only $150 \mathrm{GHz}$ ), increasing tSZ-CIB anti-correlations would increase the allowed tSZ contribution for any observed power level. However, this picture changes when we consider the other SPT frequency combinations. A tSZ-CIB anti-correlation reduces the power at $150 \times 150$ (which could be compensated by increasing tSZ power) but it also has $\sim 50 \%$ larger effect at $150 \times 220$ (where there is effectively zero tSZ power). Conversely, a tSZ-CIB anti-correlation reduces the power at $95 \times 150$ by a similar amount to $150 \times 150$, whereas there is more tSZ power in the $95 \mathrm{GHz}$ band. Adding $\mathrm{kSZ}$ power more effectively cancels the tSZ-CIB correlation term than adding tSZ power in the most sensitive combinations of the three frequencies.

Due to this degeneracy, introducing a tSZ-CIB correlation substantially degrades the constraints on both the tSZ and kSZ power. In a model with $\xi$ as a free parameter, the measured tSZ power is $D_{3000}^{\mathrm{tSZ}}=3.26 \pm 1.06 \mu \mathrm{K}^{2}$. The $95 \%$ confidence upper limit on the kSZ power is now $D_{3000}^{\mathrm{kSZ}}<6.7 \mu \mathrm{K}^{2}$. The SPT data better constrain the linear combination of $D_{3000}^{\mathrm{tSZ}}+0.5 D_{3000}^{\mathrm{kSZ}}=$ $4.60 \pm 0.63 \mu \mathrm{K}^{2}$, although the limiting case with zero tSZ and high $\mathrm{kSZ}$ power is disfavored. The expanded parameter space resembles the constraint in previous SPT work (L10, S11); the additional frequency information in this analysis is constraining the CIB model instead of breaking the degeneracy between tSZ and kSZ power. To facilitate comparison we express the constraint in the same basis as $\mathrm{S} 11$; we find a constraint of $(1.034) D_{3000}^{\mathrm{tSZ}}+0.5 D_{3000}^{\mathrm{kSZ}}=4.71 \pm 0.64 \mu \mathrm{K}^{2}$. The multiplicative factor in parentheses before the tSZ term corrects for the different effective bandcenters in the two data sets. S11 found $D_{3000}^{\mathrm{tSZ}}+0.5 D_{3000}^{\mathrm{kSZ}}=4.5 \pm 1.0 \mu \mathrm{K}^{2}$. The two measurements are completely consistent with each other. We will discuss the inferred correlation and its impact on CIB constraints in Section 9.

\subsubsection{Beyond $\Lambda C D M$}

Last, we consider whether the derived SZ and CIB powers would change with an alternate cosmological model. Current measurements of the primary CMB anisotropy contain hints 


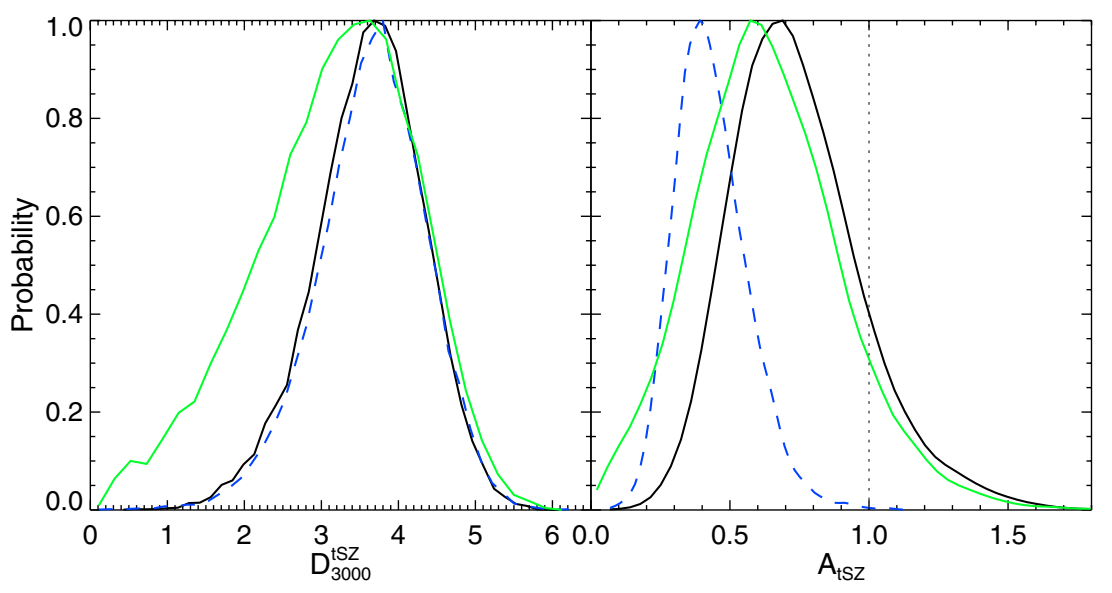

Figure 6. One-dimensional likelihood curves for the tSZ power spectra in $\mu \mathrm{K}^{2}$ at $150 \mathrm{GHz}$ and $\ell=3000$ (left panel) and ratio of measured to predicted tSZ power, $A_{\mathrm{tSZ}}$ (right panel). In the right panel, the vertical dotted line marks the expectation value for $A_{\mathrm{tSZ}}( \pm 0.04$ due to sample variance). All curves assume the CSF kSZ template, and modified BB CIB frequency scaling - the tSZ results are unaffected by these choices. The black solid line shows the results with the Shaw tSZ model. Results for the Sehgal tSZ model are shown by the blue dashed line. The power constraints (left) are identical, leading to much lower $A_{\mathrm{tSZ}}$ 's (right) as the Sehgal model predicts more tSZ power for a given cosmology. Finally, if we allow for possible correlations between the CIB and tSZ power spectra, the tSZ likelihood expands toward lower tSZ power (green line). In the correlation case, we have assumed the Shaw tSZ model—the impact is similar for the Sehgal model.

(A color version of this figure is available in the online journal.)

of parameters beyond the six $\Lambda \mathrm{CDM}$ parameters (Reichardt et al. 2009; Komatsu et al. 2011; Dunkley et al. 2011; K11). The evidence for these model extensions is not yet compelling; however, it is interesting to test whether the extended models affect the SZ constraints. We consider three extensions: running of the scalar spectral index of primordial fluctuations, massive neutrinos, and extra neutrino (or other relativistic particle) species. We find no evidence for a degeneracy between any extended parameters and the kSZ or tSZ power. The kSZ and $\mathrm{tSZ}$ constraints are unchanged under these three $\Lambda \mathrm{CDM}$ model extensions.

We also consider using an unlensed primary CMB power spectrum. We see a significant increase in the kSZ power when lensing is turned off. The inferred $\mathrm{kSZ}$ power increases by about the same amount as the primary CMB decreases in the absence of gravitational lensing at $\ell \sim 3000$. This produces a significant, $\sim 3 \mu \mathrm{K}^{2}$, shift in the $\mathrm{kSZ}$ power. However, current CMB data rule out no-lensing scenarios at more than $5 \sigma$ (K11). The induced uncertainty on the $\mathrm{kSZ}$ level for the current constraint on the lensing amplitude, $A_{\text {lens }}^{0.65}=0.95 \pm 0.15$, is only $0.4 \mu \mathrm{K}^{2}$. This is much smaller than the $1 \sigma$ uncertainty on $\mathrm{kSZ}$ and therefore can be neglected.

\section{THERMAL SZ INTERPRETATION}

We now focus on comparing our measurements of the tSZ power spectrum with the predictions of recent models and simulations. We combine measurements of the primary CMB power spectrum, $H_{0}$, and $\mathrm{BAO}$ with the $\mathrm{tSZ}$ signal to measure $\sigma_{8}$ and the sum of the neutrino masses, $\sum m_{v}$.

\subsection{Thermal SZ Constraints}

The models for the tSZ power spectrum are based on simulations with a fixed cosmology. Therefore, to correctly compare the measured $\mathrm{tSZ}$ power with theoretical predictions, we must rescale the theoretical power spectra so that they are consistent with the cosmological parameters at each point in the MCMC chains. Recall that the cosmological parameters are derived solely from constraints on $\mathrm{BAO}, \mathrm{H}_{0}$, and the primary
CMB power spectrum and so are not directly influenced by the amplitude of the measured tSZ power.

Following S11, we define a dimensionless tSZ scaling parameter, $A_{\mathrm{tSZ}}$, where

$$
A_{\mathrm{tSZ}}\left(\kappa_{i}\right)=\frac{D_{3000}^{\mathrm{tSZ}}}{\Phi_{3000}^{\mathrm{tSZ}}\left(\kappa_{i}\right)} .
$$

$D_{3000}^{\mathrm{tSZ}}$ is the measured tSZ power at $\ell=3000$ and $\Phi_{3000}^{\mathrm{tSZ}}\left(\kappa_{i}\right)$ is the template power spectrum normalized to be consistent with the set of cosmological parameters " $\kappa_{i}$ " at step $i$ in the chain. We obtain the preferred value of $A_{\mathrm{tSZ}}$ by combining the measurement of $D_{3000}^{\mathrm{tSZ}}$ with the cosmological parameter constraints provided by the primary $\mathrm{CMB}$ measurements. As described in Section 5.2, we consider two template models for the tSZ power spectrum, the "Shaw" and "Sehgal" models. These two templates bracket the range of $\mathrm{tSZ}$ models considered by $\mathrm{S} 11$.

To determine the cosmological scaling of $\Phi_{3000}^{\mathrm{tSZ}}\left(\kappa_{i}\right)$, we run the Shaw model at each step in the chain. The approximate cosmological scaling of the Shaw model around the fiducial model cosmological parameters is given by Equation (10).

In the right panel of Figure 6, we show the constraints obtained on $A_{\mathrm{tSZ}}$. The solid black line in each plot shows the results for the baseline model using the Shaw tSZ template, the CSF kSZ template, and the modified BB CIB frequency scaling, while the green line shows those obtained when a free tSZ-CIB correlation is allowed in our modeling. The blue dashed line shows the results obtained using the Sehgal tSZ model instead. The numerical constraints are also given in Table 4. Recall that a value of $A_{\mathrm{tSZ}} \simeq 1$ implies that the measured $\mathrm{tSZ}$ power is consistent with the $\mathrm{tSZ}$ template predictions, while $A_{\mathrm{tSZ}}$ less (greater) than 1 indicates that the model overestimates (underestimates) the amplitude. Note that sample variance will cause deviations from unity on the order of 0.04 .

The preferred value of $A_{\mathrm{tSZ}}$ for the Shaw template is $1.2 \sigma$ below unity (from the constraints given on $\ln A_{\mathrm{tSz}}$ ), while the preferred value of $A_{\mathrm{tSZ}}$ for the Sehgal template is $2.9 \sigma$ below unity. Although both templates predict more tSZ power than is measured, the Sehgal template is in significant tension with 
Table 4

$A_{\mathrm{tSZ}}, A_{\mathrm{kSZ}}$ Constraints

\begin{tabular}{lcccr}
\hline \hline Model & $A_{\mathrm{tSZ}}$ & $\ln A_{\mathrm{tSZ}}$ & $A_{\mathrm{kSZ}}$ & $\sigma_{8}\left[A_{\mathrm{tSZ}} \simeq 1\right]$ \\
\hline Shaw, CSF, modified BB & $0.70 \pm 0.21$ & $-0.37 \pm 0.30$ & $<1.68$ & $0.797 \pm 0.011$ \\
Sehgal, CSF, modified BB & $0.41 \pm 0.13$ & $-0.89 \pm 0.31$ & $<1.57$ & $0.767 \pm 0.009$ \\
Shaw, CSF, modified BB + tSZ-CIB & $0.60 \pm 0.24$ & $-0.54 \pm 0.41$ & $<3.98$ & $0.796 \pm 0.012$ \\
\hline
\end{tabular}

Notes. $A_{\mathrm{tSZ}}, A_{\mathrm{kSZ}}$, and $\sigma_{8}$ constraints. The distributions shown in Figure 6 are clearly non-Gaussian, therefore we give constraints on $\log A_{\mathrm{tSZ}}$ as well. Constraints on $A_{\mathrm{kSZ}}$ are $2 \sigma$ upper limits. Constraints on $\sigma_{8}$ include a prior on $\log A_{\mathrm{tSZ}}=0.00 \pm 0.04$; the width of this prior reflects sample variance but not model uncertainty. The $\mathrm{CMB}+\mathrm{BAO}+H_{0}$ constraint on $\sigma_{8}$ is $0.812 \pm 0.018$.

Table 5

Delta $\chi^{2}$ for SZ Models

\begin{tabular}{lccc}
\hline \hline tSZ Model & Homogeneous kSZ Model & $\Delta \chi^{2}$ & $\begin{array}{c}\Delta \chi^{2} \\
\text { w. X-Ray }\end{array}$ \\
\hline Shaw & CSF & $(0)$ & 0.0 \\
Shaw & NR & 2.1 & 2.3 \\
Sehgal & CSF & 8.4 & 13.0 \\
Sehgal & NR & 10.7 & 16.2 \\
\hline Free Shaw & Free CSF & -1.9 & -1.9 \\
\hline
\end{tabular}

Notes. Change to the best-fit $\chi^{2}$ when the tSZ and kSZ contributions are fixed to the predictions of different models. Differences are reported relative to the Shaw $\mathrm{tSZ} / \mathrm{CSF} \mathrm{kSZ}$ case. The model predictions are scaled to account for the specific cosmology at each chain step as described in Sections 5.2 and 5.3.1. Note that the last row has additional two free parameters describing the amplitudes of the tSZ and kSZ power spectra.

the data. As an aside, we note that using the single-SED CIB frequency scaling instead of the modified BB has little effect on the tSZ amplitude. Including a tSZ-CIB spatial correlation shifts the preferred value of $A_{\mathrm{tSZ}}$ to a slightly lower value, but principally increases the error bars (by roughly $33 \%$ ).

In Table 5, we evaluate the goodness of fit of our SZ models by running new MCMC chains in which the tSZ and kSZ terms have been fixed to their predicted, although cosmologically rescaled, values. All four combinations of the Sehgal and Shaw tSZ models and the NR and CSF kSZ models are considered. We show the change in the best-fit $\chi^{2}$ for each case relative to our baseline model (Shaw tSZ, CSF kSZ). Models that predict more SZ power are a slightly worse fit to the data. Table 4 shows that the measured tSZ power is a factor of $0.41 \pm 0.13$ times that predicted by the Sehgal template. This template predicts a tSZ amplitude that is poorly matched to the data, resulting in a $\Delta \chi^{2}=+8.4$ relative to the baseline model. Likewise, the NR kSZ template predicts $1 \mu \mathrm{K}^{2}$ more power than the CSF $\mathrm{kSZ}$ template and thus provides a worse fit to the measured powers, increasing $\chi^{2}$ by 2.1 (for Shaw tSZ) and 2.3 (Sehgal tSZ). Adding a kSZ contribution from patchy reionization would further increase the model tension.

The consistency between the SZ models and measured bandpowers can be tested further by including additional external cosmological constraints. Both the tSZ and kSZ power spectra scale most sensitively with $\sigma_{8}$. We therefore importance sample each of the MCMC chains presented in Table 5 imposing a prior of $\sigma_{8}\left(\Omega_{M} / 0.25\right)^{0.47}=0.813 \pm 0.027$, based on the X-ray cluster abundance measurements of Vikhlinin et al. (2009). The subsequent change in $\chi^{2}$ is given in the third column of the table. The prior from Vikhlinin et al. (2009) increases the significance of the discrepancy with models that predict more tSZ power. This result is particularly interesting since it eliminates one possible

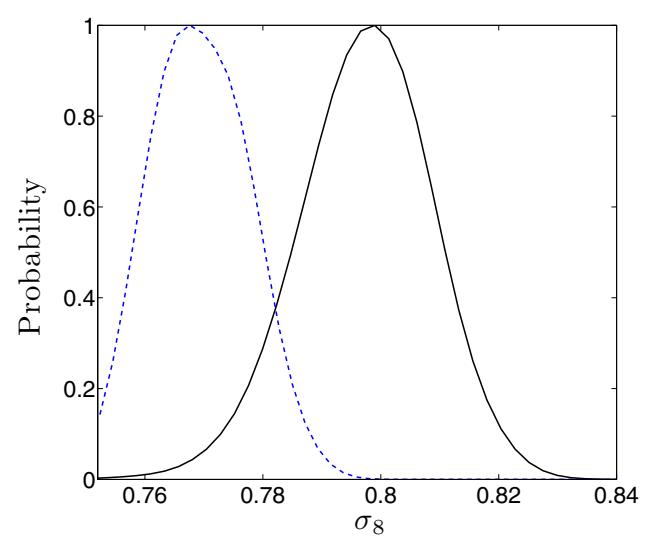

Figure 7. $\sigma_{8}$ constraints for the Shaw (black solid line) and Sehgal (blue dashed line) tSZ templates. No model uncertainty has been included.

(A color version of this figure is available in the online journal.)

solution for the high-power tSZ models-massive neutrinos. The CMB data alone are consistent with high neutrino masses which slow the growth of structure (see Section 7.3). This reduces the tSZ power predicted by all models. As a result there are cosmological parameter sets allowed at $1 \sigma$ by the CMB data, such as $\sum m_{v}=0.4 \mathrm{eV}$ and $\sigma_{8}=0.7$, for which the Sehgal model is a closer match to the observed power than the Shaw model. The X-ray constraint, $\sigma_{8} \simeq 0.8$, which is independent of the tSZ modeling, rules out this explanation.

\section{2. $\sigma_{8}$ Constraints}

To obtain joint constraints on $\sigma_{8}$ from both the primary CMB anisotropy and the tSZ power spectrum, we must determine the probability of observing the measured value of $A_{\mathrm{tSz}}$ for a given set of cosmological parameters. In the absence of sample variance - and assuming the predicted $\mathrm{tSZ}$ template to be perfectly accurate - the constraint on $\sigma_{8}$ would be given by its distribution at $A_{\mathrm{tSZ}}=1$ for each of the models. In practice, the expected sample variance of the tSZ signal is non-negligible. Based on the simulations of Shaw et al. (2009), L10 assumed the sample variance at $\ell=3000$ to be a lognormal distribution of mean 0 and width $\sigma_{\ln \left(A_{\mathrm{tsz}}\right)}=0.12$. The survey area analyzed here is eight times that of L10 and thus we reduce the width of this distribution by $\sqrt{8}$. For each of the tSZ templates, we construct new MCMC chains by importance sampling to include this prior.

The one-dimensional $\sigma_{8}$ constraints for the two tSZ templates are shown in Figure 7 and Table 4. Using the Shaw template results in $\sigma_{8}=0.797 \pm 0.011$, while the Sehgal model prefers $\sigma_{8}=0.767 \pm 0.009$. The constraint without the tSZ amplitude information (i.e., from the primary $\mathrm{CMB}+\mathrm{BAO}+\mathrm{H}_{0}$ alone) is $\sigma_{8}=0.812 \pm 0.018$. Adding the $\mathrm{tSZ}$ information therefore both 


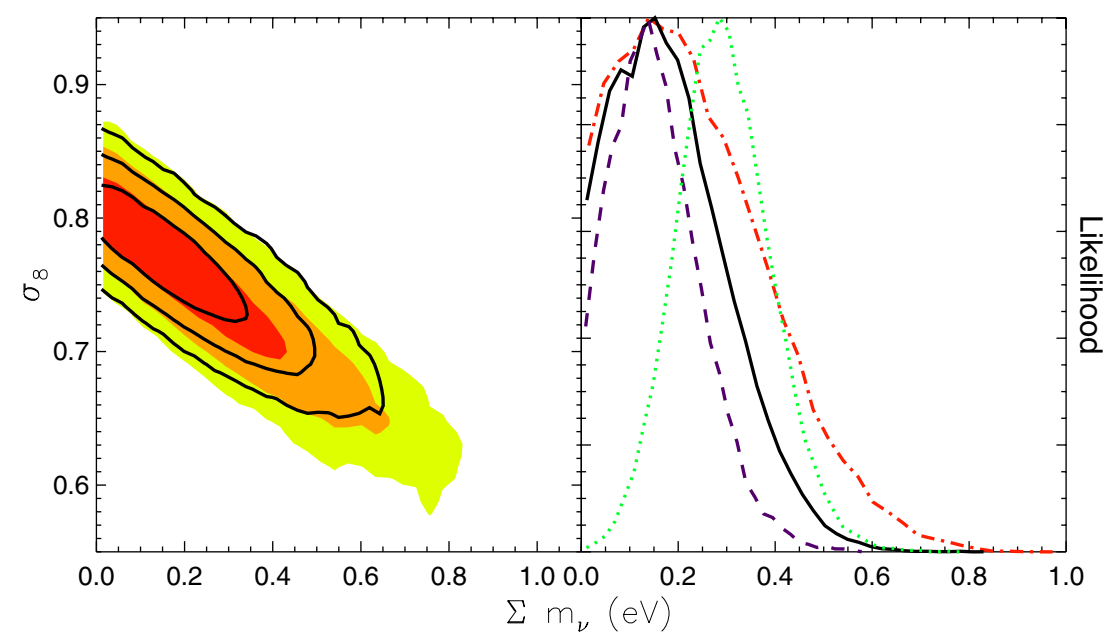

Figure 8. Left panel: two-dimensional likelihoods for $\sigma_{8}$ and $\sum m_{v}$. The filled colored contours show the $1 \sigma, 2 \sigma$, and $3 \sigma$ constraints for the CMB $+H 0+\mathrm{BAO}$ data. The black solid lines show the constraints with the addition of the tSZ information (assuming a 50\% theory prior). Right panel: one-dimensional likelihoods for the sum of neutrino masses for four cases. The red dot-dashed line shows the constraints without including tSZ data. The black solid line shows the constraints when we include the measured tSZ power with the Shaw tSZ model and a 50\% theory prior. This tightens the upper limit on the sum of the neutrino masses from $0.52 \mathrm{eV}$ to $0.40 \mathrm{eV}$ at $95 \% \mathrm{CL}$. We show the constraints on neutrino mass that would be derived with perfect tSZ modeling in the final two lines: with the Shaw tSZ model (purple dashed line) and Sehgal tSZ model (green dotted line). The Sehgal model predicts more tSZ power for a given $\sigma_{8}$. As a result, it pushes the allowed parameter volume to lower $\sigma_{8}$ and higher $\sum m_{v}$, leading to a nominal detection of the total neutrino mass at $0.29 \pm 0.10 \mathrm{eV}$.

(A color version of this figure is available in the online journal.)

reduces the size of the statistical error by $40 \%$ and lowers the preferred value, depending on the amplitude of the $\mathrm{tSZ}$ template.

The difference in the preferred value of $\sigma_{8}$ provided by the Shaw \& Sehgal templates is significantly larger than the statistical errors. This implies that the systematic error due to the theoretical uncertainty in the tSZ modeling significantly exceeds the statistical error. To produce a constraint on $\sigma_{8}$ that accounts for this, we must add an additional theory uncertainty to the sample variance when setting the prior on $A_{\mathrm{tSZ}}$.

It is difficult to determine the level of theory uncertainty on the predicted $\mathrm{tSZ}$ amplitude for a fixed set of cosmological parameters. A significant fraction of the total tSZ power is contributed by groups and clusters of mass $M_{500}<2 \times 10^{14} h^{-1} M_{\odot}$ and redshift $z>0.65$ (Trac et al. 2011; Battaglia et al. 2011b; Shaw et al. 2010). To date, such objects have not been studied in detail with SZ and X-ray observations. From a theoretical perspective, models and simulations have demonstrated that astrophysical processes such as feedback from active galactic nuclei and turbulence-driven non-thermal pressure support can significantly alter predicted cluster thermal pressure profiles, and thus their SZ signal (Battaglia et al. 2011a; Trac et al. 2011; Parrish et al. 2012; Shaw et al. 2010).

Following S11, we choose to add a $50 \%$ theory uncertainty on $\ln \left(A_{\mathrm{tSZ}}\right)$. This uncertainty encompasses all four models considered by $\mathrm{S} 11$. As the sample variance is insignificant compared to the theory uncertainty, the new prior on $A_{\mathrm{tSZ}}$ is effectively a lognormal distribution of width $\sigma\left(\ln A_{\mathrm{tSZ}}\right)=0.5$. With this assumption, we obtain constraints of $\sigma_{8}=0.807 \pm$ 0.016 for the Shaw model.

We estimate the contribution of the systematic theory uncertainty to the total uncertainty quoted above as follows. We form a new prior based on the median value of $A_{\mathrm{tSZ}}=0.7$ with the sample-variance-based width of $\sigma\left(\ln \left(A_{\mathrm{tSZ}}\right)\right)=0.042$. By sampling around the preferred value of $A_{\mathrm{tSZ}}$ instead of unity, we avoid introducing artificial tension if the modeled amplitude is incorrect. Importance sampling the MCMC chain with this prior leads to parameter constraints that include only statistical noise and sample variance. The resulting $\sigma_{8}$ con- straint is $\sigma\left(\sigma_{8}\right)=0.010$. Assuming the errors add in quadrature, this result implies a systematic modeling uncertainty of $\sigma\left(\sigma_{8}\right)=0.012$.

Given the current modeling uncertainties, the measured amplitude of tSZ power in this work only marginally improves our knowledge of $\sigma_{8}$ over existing constraints. A detailed and systematic study of the thermal pressure profiles of low-mass $\left(\approx 10^{14} h^{-1} M_{\odot}\right)$ clusters at $z \approx 0.65$ is required in order to reduce the theory uncertainty and thus increase the precision of $\sigma_{8}$ constraints derived from the tSZ power spectrum.

\section{3. $\sum m_{v}$ Constraints}

We can apply the methodology outlined above to other cosmological parameters, most notably the sum of the neutrino masses, $\sum m_{v}$. For $\mathrm{CMB}+H_{0}+\mathrm{BAO}$, neutrino masses are highly degenerate with $\sigma_{8}$ as shown in Figure 8. Higher neutrino masses lead to lower $\sigma_{8}$ since massive neutrinos slow the growth of structure below the neutrino-free-streaming length. Introducing massive neutrinos weakens the $\mathrm{CMB}+\mathrm{H}_{0}+\mathrm{BAO}$ constraint on $\sigma_{8}$ to $\sigma_{8}=0.756 \pm 0.044$. The sum of the neutrino mass is constrained to be less than $0.52 \mathrm{eV}$ at $95 \% \mathrm{CL}$.

The tSZ power spectrum presents an independent probe of $\sigma_{8}$ that breaks the $\sum m_{v}$ degeneracy and thereby improves the neutrino mass determination. The preference for positive neutrino masses increases with models that predict more tSZ power. Models that predict more tSZ power require a lower $\sigma_{8}$ to match the observed tSZ power. As can be seen in Figure 8, lower $\sigma_{8}$ values favor larger neutrino masses.

With massive neutrinos and no modeling uncertainty (but including a $4 \%$ sample variance), adding the tSZ information reduces the uncertainty on $\sigma_{8}$ by a factor of two to three. The median $\sigma_{8}$ moves up slightly to $\sigma_{8}=0.776 \pm 0.019$ with the Shaw model and down slightly to $\sigma_{8}=0.732 \pm 0.017$ with the Sehgal model. In this optimistic scenario, we would find $\sum m_{v}=0.15 \pm 0.09$ or $0.29 \pm 0.10 \mathrm{eV}$ for the Shaw and Sehgal models, respectively. Note that the X-ray $\sigma_{8}$ measurement mentioned earlier is in tension with the preferred $\sigma_{8}$ with the Sehgal model. With a more realistic $50 \%$ modeling uncertainty 


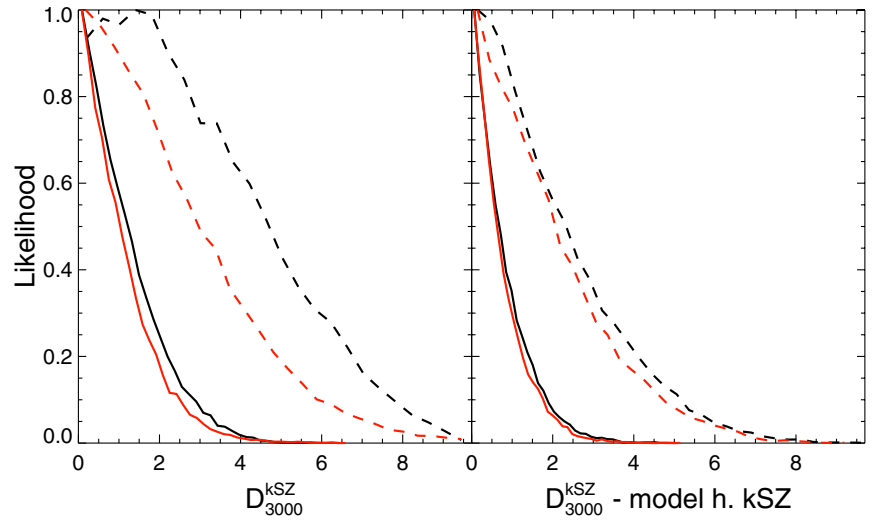

Figure 9. 1D likelihood curves for the $\mathrm{kSZ}$ power (in $\mu \mathrm{K}^{2}$ ). Left panel: constraints on the kSZ power with different CIB models and $\mathrm{kSZ}$ templates. The modified BB CIB frequency scaling and Shaw tSZ template are assumed in all cases. Solid lines denote constraints without tSZ-CIB correlations; dashed lines mark constraints with tSZ-CIB correlations allowed. Black lines show the results with the CSF kSZ template (the NR kSZ template is similar). Red lines mark the constraints when we alternatively use a template based on the kSZ contribution from the epoch of reionization. Right panel: the constraints on the patchy $\mathrm{kSZ}$ power when we include a model prediction for the homogeneous kSZ power. Solid lines denote constraints without tSZ-CIB correlations; dashed lines mark constraints with $\mathrm{tSZ}-\mathrm{CIB}$ correlations allowed. Black lines show the results when the CSF homogeneous $\mathrm{kSZ}$ model is assumed. This model includes cooling and star formation which suppresses the kSZ power. Red lines mark the constraints when the higher amplitude NR homogeneous $\mathrm{kSZ}$ model is assumed.

(A color version of this figure is available in the online journal.)

on the Shaw model, the constraint is $\sigma_{8}=0.768 \pm 0.031$. With this modeling uncertainty, the Sehgal and Shaw model results are consistent at $\sim 0.5 \sigma$. The upper limit on $\sum m_{v}$ is reduced from $0.52 \mathrm{eV}$ to $0.40 \mathrm{eV}$ at $95 \% \mathrm{CL}$ with the addition of $\mathrm{tSZ}$ information. Even with a large theory prior, the SPT tSZ measurements improve the neutrino mass constraints by disfavoring regions of parameter space with very low $\sigma_{8}$ values.

We consider how the uncertainties on $\sigma_{8}$ and $\sum m_{v}$ break down between systematic and statistical uncertainties using the method presented in Section 7.2. The prior is constructed as before and centered the preferred value of $A_{\mathrm{tSZ}}=1.14$ in the $\sum m_{v}>0 \mathrm{eV}$ chain. We find that the uncertainty on $\sigma_{8}$ can be allocated as $\sigma_{\text {sys }}\left(\sigma_{8}\right)=0.024$ (for the modeling uncertainty) and $\sigma_{\text {stat }}\left(\sigma_{8}\right)=0.020$ (for statistical noise plus sample variance). Similarly, the uncertainty on $\sum m_{v}$ can be divided into $\sigma_{\text {sys }}\left(\sum m_{v}\right)=0.10 \mathrm{eV}$ and $\sigma_{\text {stat }}\left(\sum m_{v}\right)=0.10 \mathrm{eV}$. The $50 \%$ theory uncertainty is dominant for both parameters.

\section{8. kSZ INTERPRETATION}

We next turn to comparing our measurements of the $\mathrm{kSZ}$ power spectrum with the predictions of recent models and simulations. We first compare our constraints on the amplitude of the kSZ power spectrum with our homogeneous kSZ templates. We then briefly mention the implications of the kSZ measurement for the epoch of reionization. Z11 discuss what we can learn about the epoch of reionization in more detail.

As previously presented, in the baseline model, we find the $\mathrm{kSZ}$ power to be robust to template shape between the $\mathrm{CSF}, \mathrm{NR}$, and patchy $\mathrm{kSZ}$ templates. In contrast to the tSZ, we see a small dependence on the frequency scaling of the CIB with slightly lower kSZ upper limits for the single-SED scaling than for the baseline modified BB model. However, the major modeling uncertainty, as with the tSZ, is in the tSZ-CIB correlation (see Figure 9). This correlation is highly degenerate

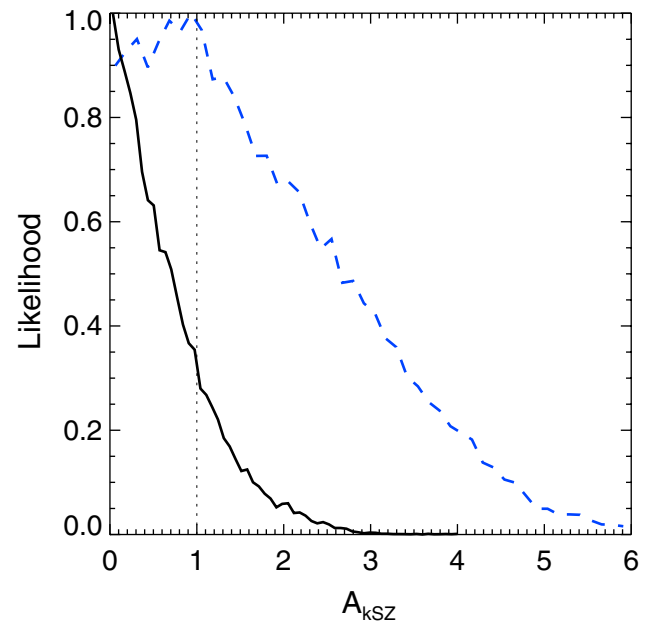

Figure 10. Posterior distribution of the kSZ scaling parameter $A_{\mathrm{kSZ}}$ (see Equation (20) and Table 4) for different CIB models. The vertical dotted line marks the model expectation value for $A_{\mathrm{kSZ}}( \pm 0.01$ due to sample variance). The CSF kSZ model we consider here is the lower of the two kSZ models and does not include the $\mathrm{kSZ}$ contribution from patchy reionization. Therefore, $A_{\mathrm{kSZ}}>1$ would be reasonable. The solid black line shows the baseline model using the Shaw tSZ template, the CSF kSZ template, and the modified BB CIB frequency scaling. The blue dashed line shows the results obtained when the tSZ-CIB correlation is allowed in our modeling.

(A color version of this figure is available in the online journal.)

with the kSZ power (see Figure 5) and, when introduced as a free parameter, nearly triples the allowed $\mathrm{kSZ} 95 \%$ upper limit. The kSZ template shape affects the kSZ upper limit at the $\sim 20 \%$ level when tSZ-CIB correlations are allowed.

\subsection{Model Comparison}

In an identical manner to the tSZ, we define a dimensionless scaling parameter $A_{\mathrm{kSZ}}$ for the homogeneous kSZ power. We use the NR and CSF kSZ models of Shaw et al. (2011). Equation (13) gives the scaling of the CSF kSZ template $\Phi_{3000}^{\mathrm{kSZ}}$ with cosmological parameters.

In Figure 10, we show the constraints obtained on $A_{\mathrm{kSZ}}$. The solid black line shows our baseline model using the Shaw tSZ template, the CSF kSZ template, and the modified BB CIB frequency scaling. The blue dashed line shows those obtained when a free tSZ-CIB correlation is included in our modeling. The numerical constraints are also given in Table 4. Recall that a value of $A_{\mathrm{kSZ}}=1$ implies that the measured $\mathrm{kSZ}$ power is consistent with the $\mathrm{kSZ}$ template predictions, while $A_{\mathrm{kSZ}}$ less (greater) than 1 indicates that the model overestimates (underestimates) the amplitude. Note that sample variance will cause deviations away from unity on the order of 0.01 .

In this work, we have significantly tightened the upper limits on the kinetic SZ power. These limits are not in significant tension with our fiducial homogeneous kSZ template (the CSF model of Shaw et al. 2011). The $2 \sigma$ upper limit on $A_{\mathrm{kSZ}}$ exceeds 1 for all of the MCMC chains in Table 4. However, for our baseline model, the upper limit is only modestly in excess of 1 . The single-SED CIB model allows for slightly less $\mathrm{kSZ}$ power than the modified BB frequency scaling. Therefore, while the data are consistent with the CSF $\mathrm{kSZ}$ prediction, there is little room for an additional contribution from patchy reionization. Z11 note that if the duration of reionization spans $\Delta z=2$ we can expect an additional $1 \mu \mathrm{K}^{2}$ of $\mathrm{kSZ}$ power. For $\Delta z=4$ we expect $2 \mu \mathrm{K}^{2}$. Clearly when combined with the post-reionization 


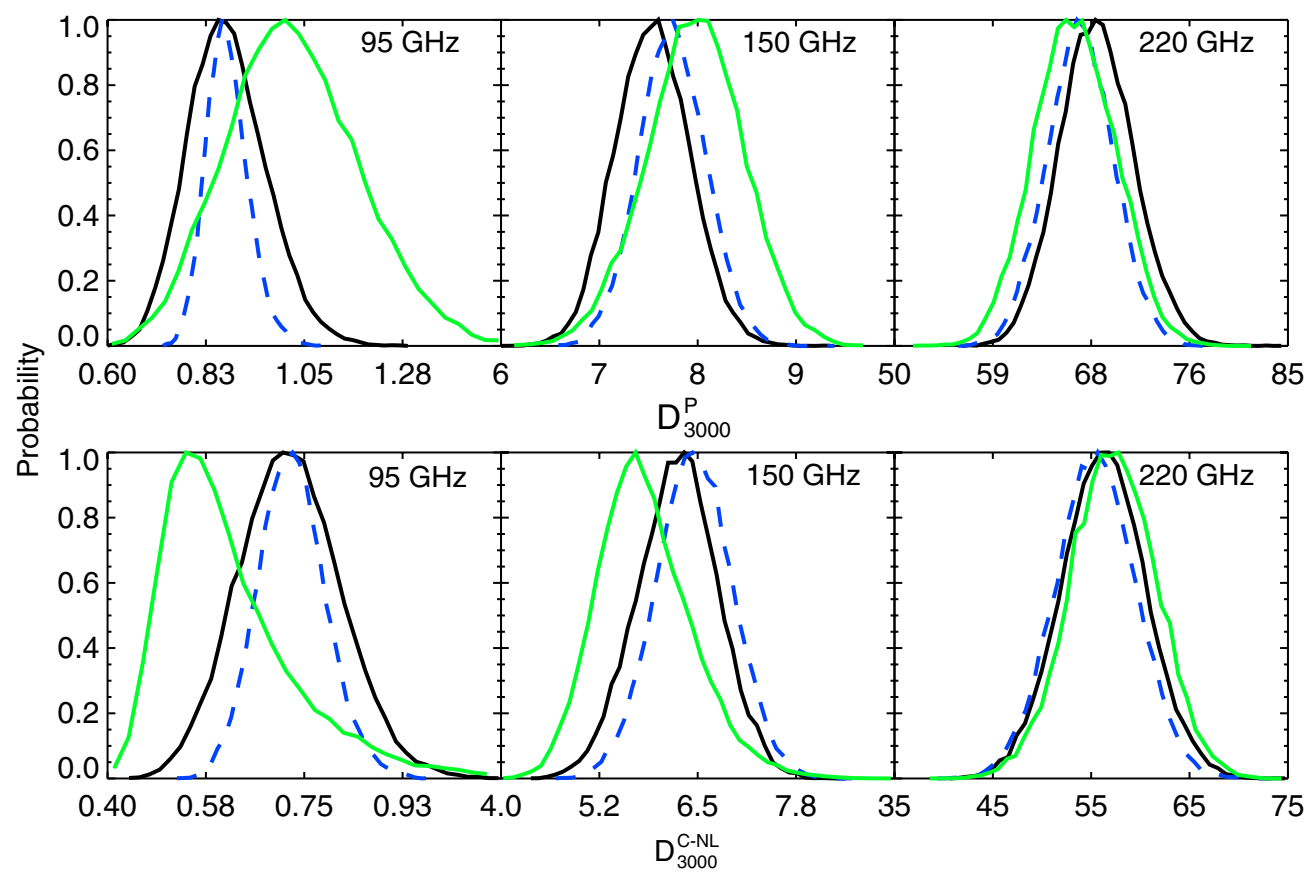

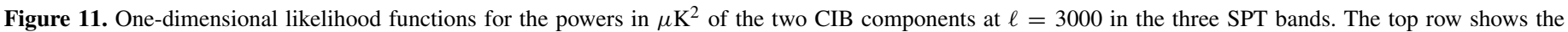

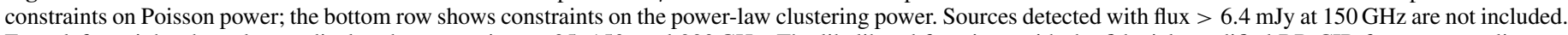

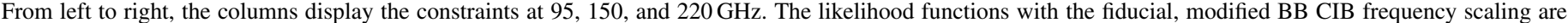

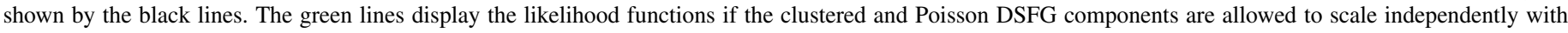

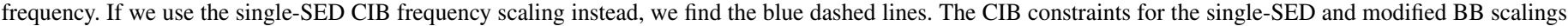

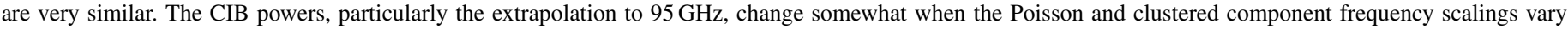

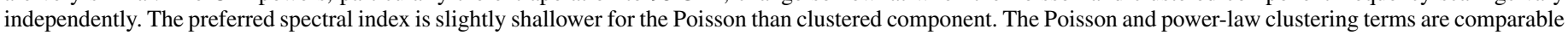
on angular scales near $\ell=2500$.

(A color version of this figure is available in the online journal.)

signal, the predicted $\mathrm{kSZ}$ will significantly exceed that measured by SPT in the baseline model.

However, as discussed in Section 6.2.1, incorporating a tSZ-CIB spatial correlation into our model allows for considerably more $\mathrm{kSZ}$ power than our fiducial model. In this case, the upper limit on $A_{\mathrm{kSZ}}$ is 4.13 , which allows for substantially more power than predicted by the homogeneous kSZ models alone, and thus a significant contribution from patchy reionization.

\subsection{Patchy Reionization}

We now switch to considering limits on the kSZ signal contributed by patchy reionization. For this we use a patchy kSZ template from Z11 based on a model in which reionization started at $z=11$ and ended $z=8$. As would be expected, the kSZ signals from the reionization and post-reionization era are nearly perfectly degenerate. Therefore, our constraints on the patchy $\mathrm{kSZ}$ power depend on the modeling of the postreionization homogeneous $\mathrm{kSZ}$ power. We choose to bracket the latter with three cases: no homogeneous kSZ, the intermediate CSF homogeneous kSZ model, and the highest NR homogeneous kSZ model.

The constraints on patchy kSZ power are shown in Figure 9. Note that we see less difference in the allowed patchy kSZ power than would be inferred from the power differences between the assumed homogeneous kSZ model. The smaller-than-expected differences are because the likelihood function for the total $\mathrm{kSZ}$ power, before any amount of homogeneous $\mathrm{kSZ}$ power is subtracted, already peaks at zero. We find 95\% CL upper limits on the kSZ power due to patchy reionization of 2.6 , 2.0, and $1.9 \mu \mathrm{K}^{2}$ for the zero, CSF, and NR homogeneous
$\mathrm{kSZ}$ models, respectively. As with all the kSZ constraints, we find the patchy kSZ constraints are most sensitive to tSZ-CIB correlations. With a free tSZ-CIB correlation and the same set of three homogeneous kSZ models, the 95\% CL upper limits on patchy $\mathrm{kSZ}$ power are degraded to $5.7,4.9$, and $4.7 \mu \mathrm{K}^{2} . \mathrm{Z} 11$ interpret these upper limits on patchy $\mathrm{kSZ}$ power to constrain the duration of the epoch of reionization and the ionization history of the universe.

\section{COSMIC INFRARED BACKGROUND CONSTRAINTS}

The combination of Planck/HFI and SPT data strongly constrains the CIB power spectrum at millimeter wavelengths, especially at $220 \mathrm{GHz}$. The CIB constraints for a variety of model assumptions are summarized in Table 6 and Figure 11. Recall that, in all cases, sources with flux $>6.4 \mathrm{mJy}$ at $150 \mathrm{GHz}$ have been masked. We find the $220 \mathrm{GHz}$ Poisson amplitude at $\ell=3000$ to be $D_{3000}^{p}=68.0 \pm 3.3 \mu \mathrm{K}^{2}$. The clustering amplitude is $D_{3000}^{c}=56.3 \pm 4.2 \mu \mathrm{K}^{2}$-about $45 \%$ of the total CIB power at this angular scale. The cross-over point where the Poisson and clustering terms have equal power is at $\ell=2500$. At $150 \mathrm{GHz}$, the Poisson and clustering powers are $D_{3000}^{p}=7.54 \pm 0.38 \mu \mathrm{K}^{2}$ and $D_{3000}^{c}=6.25 \pm 0.52 \mu \mathrm{K}^{2}$, respectively. The estimated $\mathrm{CIB}$ at $95 \mathrm{GHz}$ is very small, but this is an extrapolation from higher frequencies and sensitive to the frequency modeling. Both Poisson and clustered CIB components have more power at $150 \mathrm{GHz}$ than the SZ spectra across the multipoles $(\ell>2000)$ measured in this work. We will leverage the wide range of angular scales probed by SPT and Planck/HFI to constrain the shape of the CIB clustering in Section 9.1. 
Table 6

CIB Constraints

\begin{tabular}{|c|c|c|c|c|c|c|}
\hline \multirow[t]{2}{*}{ Model } & \multicolumn{3}{|c|}{ Poisson $\left(\mu \mathrm{K}^{2}\right)$} & \multirow[t]{2}{*}{$\alpha_{p}^{150-220}$} & \multirow[t]{2}{*}{$\alpha_{c}^{150-220}$} & \multirow[t]{2}{*}{ tSZ-CIB Correlation } \\
\hline & $95 \mathrm{GHz}$ & $150 \mathrm{GHz}$ & $220 \mathrm{GHz}$ & & & \\
\hline Mod. BB & $0.87 \pm 0.09$ & $7.54 \pm 0.38$ & $67.96 \pm 3.29$ & $3.56 \pm 0.07$ & $\ldots$ & $\ldots$ \\
\hline$\alpha_{c} \neq \alpha_{p}$ & $1.02 \pm 0.15$ & $7.97 \pm 0.49$ & $65.99 \pm 3.65$ & $3.45 \pm 0.11$ & $3.72 \pm 0.12$ & $\ldots$ \\
\hline Linear-theory clustering & $0.90 \pm 0.10$ & $7.73 \pm 0.48$ & $69.10 \pm 3.88$ & $3.55 \pm 0.08$ & $\ldots$ & $\ldots$ \\
\hline Power law & $0.86 \pm 0.10$ & $7.50 \pm 0.52$ & $67.79 \pm 4.23$ & $3.57 \pm 0.08$ & $\ldots$ & $\ldots$ \\
\hline tSZ-CIB correlation & $1.04 \pm 0.15$ & $8.05 \pm 0.48$ & $66.67 \pm 3.68$ & $3.45 \pm 0.11$ & $\ldots$ & $-0.18 \pm 0.11$ \\
\hline \multirow[t]{3}{*}{ Single Sed } & $0.87 \pm 0.05$ & $7.74 \pm 0.34$ & $66.51 \pm 2.96$ & $3.51 \pm 0.04$ & $\ldots$ & $\ldots$ \\
\hline & \multicolumn{3}{|c|}{ Non-linear clustering $\left(\mu \mathrm{K}^{2}\right)$} & \multicolumn{3}{|c|}{ Linear theory clustering $\left(\mu \mathrm{K}^{2}\right)$} \\
\hline & $95 \mathrm{GHz}$ & $150 \mathrm{GHz}$ & $220 \mathrm{GHz}$ & $95 \mathrm{GHz}$ & $150 \mathrm{GHz}$ & $220 \mathrm{GHz}$ \\
\hline Model BB & $0.72 \pm 0.09$ & $6.25 \pm 0.52$ & $56.26 \pm 4.22$ & $\ldots$ & $\ldots$ & $\ldots$ \\
\hline$\alpha_{c} \neq \alpha_{p}$ & $0.59 \pm 0.11$ & $5.77 \pm 0.59$ & $57.26 \pm 4.34$ & $\ldots$ & $\ldots$ & $\ldots$ \\
\hline Linear-theory clustering & $0.67 \pm 0.13$ & $5.73 \pm 1.00$ & $51.13 \pm 9.12$ & $0.03 \pm 0.05$ & $0.26 \pm 0.43$ & $2.39 \pm 3.78$ \\
\hline Power law & $0.72 \pm 0.11$ & $6.28 \pm 0.71$ & $56.70 \pm 6.39$ & $\ldots$ & $\ldots$ & $\ldots$ \\
\hline tSZ-CIB correlation & $0.87 \pm 0.17$ & $6.73 \pm 0.73$ & $55.85 \pm 4.47$ & $\ldots$ & $\ldots$ & $\ldots$ \\
\hline Single Sed & $0.73 \pm 0.06$ & $6.46 \pm 0.48$ & $55.48 \pm 4.10$ & $\ldots$ & $\ldots$ & $\ldots$ \\
\hline
\end{tabular}

Notes. Constraints on the CIB power and spectral index are presented for six CIB models. Five of these models use the modified BB frequency dependence for the CIB. The first row is for the baseline model. The next four rows consider one-parameter extensions to the baseline CIB treatment. In the " $\alpha_{c} \neq \alpha_{p}$ " row, the Poisson and clustered terms have independent frequency scalings. In the "Linear-theory clustering" row, we allow for a (positive or negative) linear theory clustering term in addition to the power-law clustering term. In the "power-law" row, we introduce the exponent of the power-law clustering term as a free parameter instead of fixing it to 0.8. In the "tSZ-CIB correlation" row, we allow for correlations between the tSZ and CIB components. The last row considers constraints under the alternate single-SED frequency modeling for the CIB. For each model, we present the constraints on the amplitudes in $\mu \mathrm{K}^{2}$ at $\ell=3000$ of the Poisson and power-law clustering CIB templates. For the linear-theory clustering row, we also present the preferred amplitudes of the linear-theory term-none of which represent detections. Finally, we present the effective spectral index from 150 to $220 \mathrm{GHz}$ for the Poisson (and if different) the clustering terms.

Both models considered for the frequency scaling of the CIB lead to similar results. The data require a steep dropoff in CIB power from 220 to $150 \mathrm{GHz}$; the spectral index is $\alpha_{150 / 220}=3.51 \pm 0.04$ and $\alpha_{150 / 220}=3.56 \pm 0.07$ for the single SED and modified BB models, respectively. This is shallower by $1 \sigma$ than the $\alpha_{150 / 220}=3.68 \pm 0.07$ measured for the clustered spectral index by Addison et al. (2012) using data from BLAST, Planck/HFI, and ACT. We note that when we allow the Poisson and clustered spectral indices to differ later in this section, the inferred clustered spectral index becomes more consistent with the Addison et al. (2012) result. The observed spectral index for the lowest two published Planck/HFI channels $(217$ and $353 \mathrm{GHz})$ is shallower: $\alpha_{217 / 353}=$ $3.22 \pm 0.08$.

These indices are steeper than many current models predict. For instance, the best-fit parameters found by Fixsen et al. (1998) when fitting COBE-FIRAS data predict $\alpha_{150 / 220}=2.6$. The Planck Collaboration et al. (2011) previously pointed out that these parameters also overpredict the CIB anisotropy from 217 to $857 \mathrm{GHz}$. The alternate parameter fitting by Gispert et al. (2000) that works well across the Planck/HFI frequencies (Planck Collaboration et al. 2011) still predicts a shallower $\alpha_{150 / 220}=3.04$ between 150 and $220 \mathrm{GHz}$. Béthermin et al. (2011) modeled DSFG source counts using data from IRAS, Spitzer, Herschel, and AzTEC - these modeled source counts were used to predict the Poisson power in the Planck/HFI CIB bandpowers (Planck Collaboration et al. 2011). The spectral index predicted by the Béthermin model is $\alpha_{150 / 220}=2.8$. The maximum spectral index among the ten provided realizations of the Béthermin model is $\alpha_{150 / 220}=3.1$. These models are a poor fit to the observed CIB power at millimeter wavelengths. For instance, the Béthermin model predicts $\sim 25 \%$ too much Poisson power at $220 \mathrm{GHz}$ and twice the observed power at $150 \mathrm{GHz}$.
There is a clear degeneracy between the two parameters of the modified BB model with only $\leqslant 220 \mathrm{GHz}$ data. Recall that in this model, the CIB spectrum is described by $v^{\beta} B_{v}(T)$. Without $353 \mathrm{GHz}$ data, temperatures above $\sim 15 \mathrm{~K}$ are constrained only by the prior since the observed frequencies are in the RJ tail of the spectrum. The preferred temperature is $>12 \mathrm{~K}$ and $\beta=1.8 \pm 0.1$. These results are plotted in Figure 12. Adding $353 \mathrm{GHz}$ data partly breaks the $T-\beta$ degeneracy. We showed earlier in Section 5.4.3 that the spectral index was shallower in the higher frequency Planck/HFI data $-\alpha_{217 / 353}=3.22 \pm 0.08$. The changing slope means that the modified BB model prefers lower temperatures $(16 \pm 6 \mathrm{~K}$, with a non-Gaussian distribution) and high values of $\beta(\beta=1.80 \pm 0.14)$ when we include the $353 \mathrm{GHz}$ data. The modified BB parameters, with and without $353 \mathrm{GHz}$ data, are consistent at $<1 \sigma$.

With the single-SED frequency scaling, the median $z_{\mathrm{c}}=$ 0.53 , but the best-fit $z_{\mathrm{c}} \sim 0$. Recall that in the single-SED model, the DSFG density as a function of redshift is described by a Gaussian centered at $z_{\mathrm{c}}$. The distribution of $z_{\mathrm{c}}$ tightens around zero when the $353 \mathrm{GHz}$ data are added. This suggests that either the adopted SED template is not representative or that using a single SED for all galaxies is an oversimplification. Note that in the single-SED model, the maximum allowed spectral index is $\alpha=3.57$ at $z_{\mathrm{c}}=0$. For the specified model parameters, higher spectral indices would require $z_{\mathrm{c}}<0$, which is not allowed by the $z_{\mathrm{c}}$ prior. The data are exploring the area at this limit, which slightly lowers the median spectral index for the singleSED model. These redshifts are driven low to accommodate the steep observed spectral index. These constraints are shown in Figure 12. The tighter spectral index range with the single-SED model also (artificially) tightens the kSZ constraints by a small amount.

We explore scaling the clustered and Poisson CIB independently with frequency, and find a small preference for different 

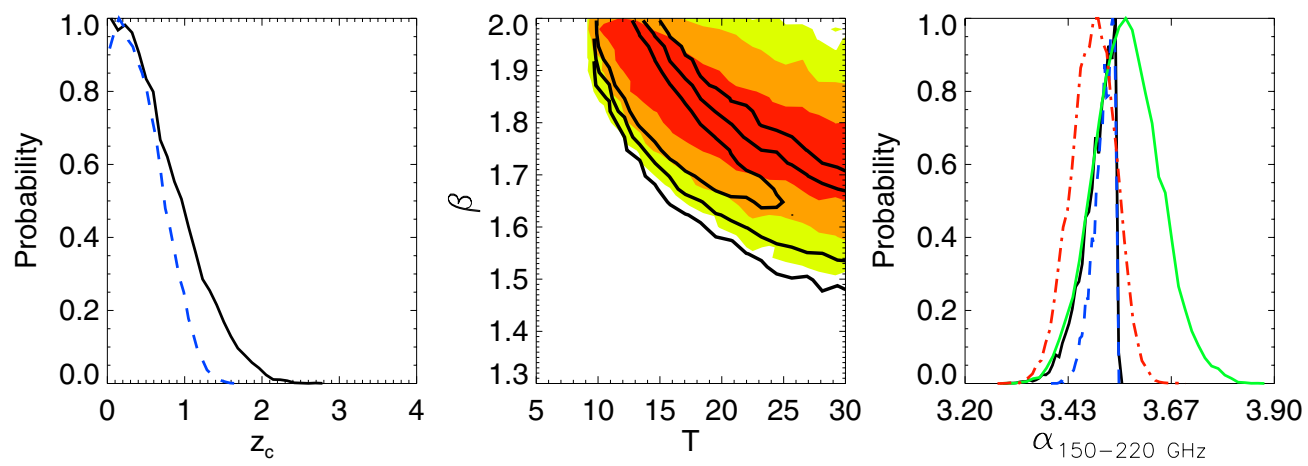

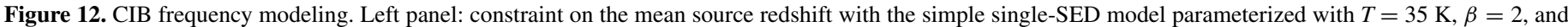

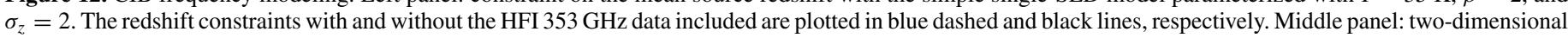

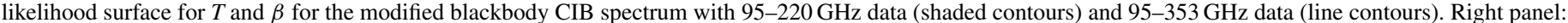

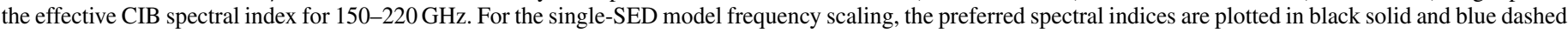

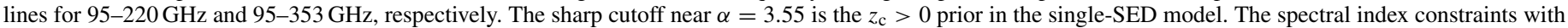
the modified blackbody model with and without $353 \mathrm{GHz}$ are shown by the red dot-dashed and green solid lines, respectively.

(A color version of this figure is available in the online journal.)

spectral indices when considering only $\leqslant 220 \mathrm{GHz}$ data. For instance, with the modified BB model, the $150-220 \mathrm{GHz}$ spectral index is $\alpha_{150 / 220}=3.45 \pm 0.11$ and $3.72 \pm 0.12$ for the Poisson and clustered term, respectively-a difference of $1.7 \sigma$. The spectral index constraint is modestly weakened by the additional free parameter, but both parameters remain well constrained. The SZ constraints are not affected. However, we note that adding the $353 \mathrm{GHz}$ Planck/HFI data removes this small preference for different spectral indices. In this case, the Poisson and clustered spectral indices are $\alpha_{150 / 220}=3.46 \pm 0.09$ and $3.50 \pm 0.08$, respectively.

\subsection{Angular Dependence of the Clustered CIB Component}

As noted earlier, we find strong evidence for clustered DSFGs in the data. We have modeled the clustering by a power law with $D_{\ell} \propto \ell^{0.8}$; here we examine if the data prefer an alternate shape. The SPT data alone can be fit with either a linear-theory clustering template or the power-law baseline template-only the extremely wide angular range probed by the combination of SPT and HFI allows shape discrimination in the clustered template. We parameterize the CIB clustering shape in two ways: fitting for the exponent of the power law, or fitting for the amplitude of a linear-theory term in addition to the powerlaw term (with fixed exponent). Adding linear theory power is similar in effect to lowering the power-law exponent.

We introduce the power-law exponent, $\gamma$, as a new parameter to the MCMC parameter chains. A uniform prior is imposed from 0.1 to 1.4 . The upper edge is chosen to prevent overlap with the $\ell^{2}$ scaling of the Poisson term. The constraints on $\gamma$ are shown in Figure 13. The data prefer $\gamma=0.81 \pm 0.08$, consistent with the nominal value of 0.8 . Adding the Planck/HFI $353 \mathrm{GHz}$ CIB bandpowers improves the shape measurement to $\gamma=0.82 \pm 0.05$. The likelihood of the best-fit point is not improved by the introduction of this new parameter for either set of data. Both results are consistent with the recent constraint of $\gamma=0.75 \pm 0.06$ by Addison et al. (2012) using data from BLAST, Planck/HFI, and ACT. For simplicity and ease of comparison with earlier results, we continue to use $\gamma=0.8$ in all other parameter chains.

We also test introducing the linear theory model with a free amplitude in addition to the power-law term with $\gamma=0.8$. The constraints on the amplitudes of the linear theory and power-law terms at $220 \mathrm{GHz}$ and $\ell=3000$ are shown in Figure 14. We

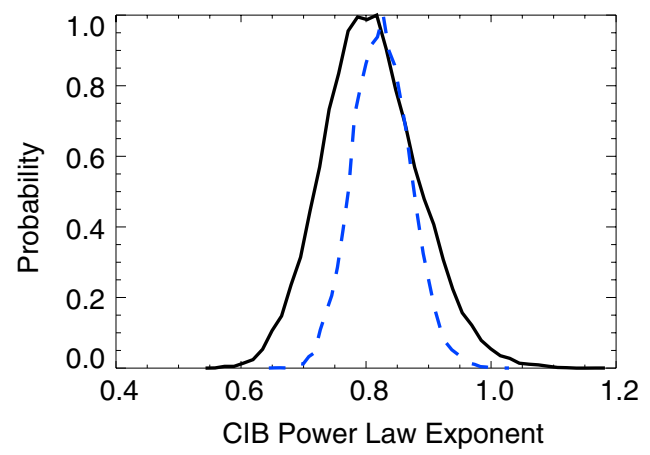

Figure 13. One-dimensional likelihood function for the power-law exponent, $\gamma$, of the CIB clustering term $\left(D_{\ell} \propto \ell^{\gamma}\right)$. The fiducial model value is 0.8 . The curves for constraints with and without including the HFI $353 \mathrm{GHz}$ CIB bandpowers are plotted with dashed blue and black lines, respectively.

(A color version of this figure is available in the online journal.)

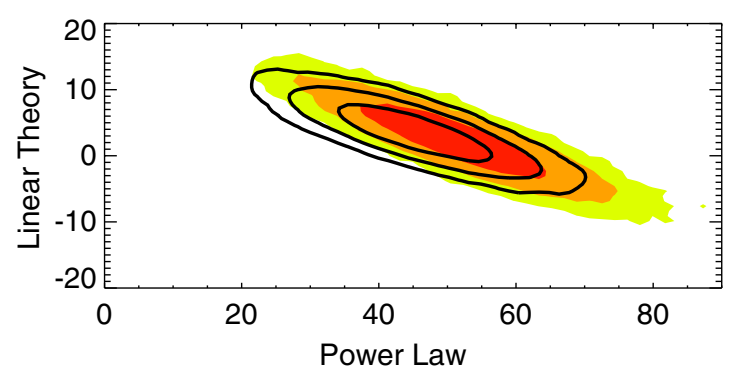

Figure 14. Two-dimensional likelihood function for the amplitudes in $\mu \mathrm{K}^{2}$ at $220 \mathrm{GHz}$ of the power-law and linear-theory CIB clustering terms. The filled yellow to red contours show the $1 \sigma, 2 \sigma$, and $3 \sigma$ constraints with SPT + Planck/ HFI data at $95-220 \mathrm{GHz}$. The black contours show the same constraints when including the Planck/HFI $353 \mathrm{GHz}$ CIB bandpowers. In both cases, the data significantly prefer the power-law clustering template and are consistent at $1 \sigma$ with zero additional linear theory power.

(A color version of this figure is available in the online journal.)

do not find a significant preference for a non-zero amplitude of the linear theory term; the derived $1 \sigma$ confidence intervals are $\left[-0.02,0.08 \mu \mathrm{K}^{2}\right],\left[-0.2,0.7 \mu \mathrm{K}^{2}\right]$, and $\left[-1.4,6.2 \mu \mathrm{K}^{2}\right]$ at 95,150 , and $220 \mathrm{GHz}$, respectively. We have allowed negative values to allow the maximum shape flexibility; the total power in the two clustering terms is always positive. Note that these ranges should be compared to $0.67 \pm 0.13,5.7 \pm 1.0$, and 
$51 \pm 9 \mu \mathrm{K}^{2}$ for the corresponding amplitude of the power-law term. The quality of the fit is not improved by adding this free parameter. The preferred values imply that most clustered CIB power is in the power-law shape instead of the linear theory shape at $\ell \gtrsim 500$. Adding the Planck/HFI $353 \mathrm{GHz}$ bandpowers does not significantly change this picture.

\subsection{CIB Constraints with $t S Z-C I B$ Correlation}

The galaxy clusters that contribute to the tSZ power spectrum have a higher than average density of galaxies, each with some amount of star formation. This will lead to an anti-correlation between the tSZ and CIB power spectra. We introduce this correlation following the treatment in Section 5.4.4. Note that in this treatment, the correlation is defined with respect to all CIB power, although the Poisson and clustered components may correlate differently. As discussed in Z11, some models predict a correlation effectively independent of $\ell$ at current instrumental sensitivities (e.g., Sehgal et al. 2010), while others predict that the magnitude of the correlation will rise across the relevant multipoles. We use a single correlation coefficient on all angular scales. The data prefer a correlation coefficient of $\xi=-0.18 \pm 0.12$ (i.e., an anti-correlation between tSZ and CIB signals, consistent with a fraction of the CIB emission being spatially coincident with the tSZ decrement from clusters). This constraint depends on the assumption that the correlation is independent of $\ell$. We tested a correlation model described by Z11 where the absolute magnitude of the correlation doubles from $\ell=2000$ to 10,000 and found similar uncertainties but a mean value $1 \sigma$ closer to zero at $\ell=3000$. In the near future, we expect observations with Herschel and Planck in combination with SPT to place better observational constraints on this correlation.

Introducing a tSZ-CIB correlation does very little to the inferred CIB powers; however, the uncertainties increase by 10\%-50\%. With tSZ-CIB correlations, the constraint on the Poisson power at $150 \mathrm{GHz}$ is $D_{3000}^{p}=8.04 \pm 0.48 \mu \mathrm{K}^{2}$, and the constraint on the clustered power at $150 \mathrm{GHz}$ is $D_{3000}^{c}=$ $6.71 \pm 0.74 \mu \mathrm{K}^{2}$. The best-fit spectral index is lower by $1 \sigma$, and the uncertainty increases by $50 \%$ to give $\alpha=3.45 \pm 0.11$. However as discussed earlier, a free correlation significantly degrades the kSZ constraint.

\section{CONCLUSIONS}

We have presented the CMB temperature anisotropy power spectrum from the complete $800 \mathrm{deg}^{2}$ of sky observed by SPT in the 2008 and 2009 austral winters. The six cross-spectra from observations at three frequencies $(95,150$, and $220 \mathrm{GHz})$ are shown in Table 1 . The bandpowers measure arcminute-scale $(\ell>2000)$ millimeter wavelength anisotropy. On these small scales, DSFGs, radio galaxies, and the kinetic and thermal SZ effects contribute significant power in addition to the primary CMB anisotropy.

Using MCMC methods, we perform multi-frequency fits to a combined data set including WMAP7, BAO, $H_{0}$, previously published low- $\ell$ SPT bandpowers, Planck/HFI CIB bandpowers, and the $\ell>2000$ SPT bandpowers presented in this work. We find that the minimal model needed to explain the current data adds five free parameters beyond the six $\Lambda$ CDM parameters: four describing DSFGs and one describing the amplitude of the tSZ power spectrum. We also include a free parameter for the amplitude of the $\mathrm{kSZ}$ spectrum and a prior on a Poisson population of radio galaxies. This six parameter extension im- proves the likelihood of the best-fit model by $\Delta \chi^{2}=-2081$. We explore a number of extensions to this baseline model, including different templates for the $\mathrm{kSZ}$, tSZ, or CIB terms, different modeling of the CIB frequency spectrum, and correlations between the tSZ and CIB. We do not find that these extensions significantly improve the quality of fits to the data, however, they may slightly alter the conclusions drawn.

We fit for the amplitude of the tSZ and $\mathrm{kSZ}$ power spectra, using a single amplitude parameter defined in units of $\mu \mathrm{K}^{2}$ at $\ell=3000$ and $150 \mathrm{GHz}$. The constraint on the $150 \mathrm{GHz}$ tSZ power is $D_{3000}^{\mathrm{tSZ}}=3.65 \pm 0.69 \mu \mathrm{K}^{2}$ in the baseline model. We find that the tSZ amplitude is independent of the template shape for the two models considered: the Shaw and Sehgal tSZ models. We find that the kSZ results are also robust with respect to template shape. The $95 \%$ confidence upper limit on the $\mathrm{kSZ}$ power is $D_{3000}^{\mathrm{kSZ}}<2.8 \mu \mathrm{K}^{2}$.

We test whether extensions to the primary CMB modeling, including running of the scalar index, changing number of neutrino species, or massive neutrinos, affect the SZ constraints and find they do not. We also investigate whether alternative CIB modeling assumptions lead to changes in the SZ constraints. The SZ constraints are fairly insensitive to the CIB modeling. An exception is their sensitivity to the modeling of the tSZ-CIB correlation. When allowing for the possibility of this correlation, the allowed parameter space expands along the degeneracy line of $D_{3000}^{\mathrm{tSZ}}+0.5 D_{3000}^{\mathrm{kSZ}}$. The intersection of this line moves by less than $1 \sigma: D_{3000}^{\mathrm{tSZ}}+0.5 D_{3000}^{\mathrm{kSZ}}=4.15 \pm 0.56 \mu \mathrm{K}^{2}$ without correlations and $4.60 \pm 0.63 \mu \mathrm{K}^{2}$ with free correlations. We note that this degeneracy line closely resembles that found by L10, who used a linear combination of the $150 \mathrm{GHz}$ and $220 \mathrm{GHz}$ maps to subtract the CIB. When correlations are allowed, the $95 \%$ confidence upper limits on the total $\mathrm{kSZ}$ power are $D_{3000}^{\mathrm{kSZ}}<6.7 \mu \mathrm{K}^{2}$ and $D_{3000}^{\mathrm{kSZ}}<5.7 \mu \mathrm{K}^{2}$ for the homogeneous and patchy reionization templates, respectively. The real $\mathrm{kSZ}$ signal is expected to be an admixture of the two templates.

We use the measured tSZ power to constrain $\sigma_{8}$ and the sum of the neutrino masses. For massless neutrinos and the optimistic assumption of assuming perfect tSZ modeling, the tSZ power constraint tightens the $\mathrm{CMB}+\mathrm{BAO}+\mathrm{H}_{0} \sigma_{8}$ constraint by $40 \%$. However, the preferred $\sigma_{8}$ value is $3 \sigma$ discrepant between the two (Shaw and Sehgal) tSZ models considered. With a conservative $50 \%$ modeling uncertainty, adding the tSZ information does not significantly improve the constraints on $\sigma_{8}$. The impact of the tSZ power measurement is more significant when neutrinos are allowed to have mass. With massive neutrinos, even with a $50 \%$ modeling uncertainty, the tSZ power spectrum reduces the $\sigma_{8}$ uncertainty by $30 \%$ and tightens the upper limit on the total neutrino mass from $0.52 \mathrm{eV}$ to $0.40 \mathrm{eV}$. Precise measurements of the pressure profiles of high-redshift clusters are essential to reduce the theory uncertainty and realize the statistical power of the tSZ measurement.

We also use the SPT data to constrain the astrophysical foregrounds (after masking the brightest point sources). Two parameters - which can be fixed with priors from other observations - fit the radio source component. We find that a four-parameter model is adequate to describe the Poisson and clustered DSFGs. The spectrum of the DSFGs falls sharply from 220 to $150 \mathrm{GHz}$, with an effective spectral index of $\alpha=3.56 \pm 0.07$. This is steeper by $\sim 2 \sigma$ than extrapolations from models based on higher frequency observations of the CIB. However, it is in agreement with previous spectral index measurements at these frequencies by S11, Dunkley et al. 
(2011), and Addison et al. (2012). We find Poisson DSFG powers at $\ell=3000$ of $D_{3000}^{p}=7.54 \pm 0.38 \mu \mathrm{K}^{2}$ and $D_{3000}^{p}=$ $68.0 \pm 3.3 \mu \mathrm{K}^{2}$ at 150 and $220 \mathrm{GHz}$, respectively. We find clustered DSFG powers at $\ell=3000$ of $D_{3000}^{c}=6.25 \pm 0.52 \mu \mathrm{K}^{2}$ and $D_{3000}^{c}=56.3 \pm 4.2 \mu \mathrm{K}^{2}$ at 150 and $220 \mathrm{GHz}$, respectively. We find some preference for different spectral indices for the clustering and Poisson DSFG components when we exclude the Planck/HFI $353 \mathrm{GHz}$ data; this preference vanishes when the $353 \mathrm{GHz}$ data are included.

With Planck/HFI and SPT data, the CIB is constrained on angular scales from $\ell=80$ to 9400 at $220 \mathrm{GHz}$. This large lever arm allows both the separation of the Poisson and clustered DSFG components, and a tight constraint on the shape of the clustering component. We find that the data prefer the clustered power to be predominately in the form of a power-law $\ell^{\gamma}$ rather than the linear theory prediction. This is strong evidence for nonlinearities in the DSFG clustering. We further constrain the exponent of the power law to be $\gamma=0.82 \pm 0.05$, consistent with other recent constraints (Addison et al. 2012).

We test the impact of introducing a tSZ-CIB correlation coefficient and find that the data prefer a negative correlation at $98 \%$ confidence. Note that this significance may vary for more sophisticated CIB models. The tSZ-CIB anti-correlation modestly increases the inferred CIB powers, while counterintuitively reducing the $\mathrm{tSZ}$ power and increasing the allowed $\mathrm{kSZ}$ power. The uncertainties on both SZ terms increase significantly when correlations are allowed. External data or modeling to constrain the $\mathrm{tSZ}-\mathrm{CIB}$ correlation would be extremely valuable to future $\mathrm{kSZ}$ constraints.

A companion paper, Z11, uses the SPT bandpowers and kSZ upper limits presented here to investigate the epoch of reionization. Z11 constrains the duration of reionization from the SPT measurement of kSZ power. The midpoint of reionization is determined from the WMAP constraint on the optical depth. Together, the measurements yield the first constraints on the beginning, duration, and end of reionization from the CMB.

The SPT survey of $2500 \mathrm{deg}^{2}$ finishes in 2011 November. The survey area, comprising $6 \%$ of the total sky, has been mapped to depths of approximately 40,18 , and $70 \mu \mathrm{K}$ arcmin at 95,150 , and $220 \mathrm{GHz}$, respectively. We expect bandpower uncertainties for the full data set to be reduced by a factor of 1.7 compared to this work. We expect from simulations that the constraints on the tSZ, kSZ, and tSZ-CIB correlation will improve commensurately. In addition, we have selected $100 \mathrm{deg}^{2}$ of the SPT survey for deeper integration and Herschel followup. Herschel/SPIRE will observe this region in the winter of 2011. The combined SPT/Herschel data set will be an invaluable resource in understanding the CIB and its correlation with the SZ power spectra. We will be able to use the Herschel maps to clean the CIB from the SPT maps of this $100 \mathrm{deg}^{2}$ field, further improving measurements of the tSZ and kSZ power spectra. These results will advance our understanding of structure formation and the reionization of the universe.

We thank Cien Shang, Guilaine Lagache, Olivier Dore, and Marco Viero for useful discussions and assistance with the CIB modeling. The South Pole Telescope is supported by the National Science Foundation through grants ANT-0638937 and ANT-0130612. Partial support is also provided by the NSF Physics Frontier Center grant PHY-0114422 to the Kavli Institute of Cosmological Physics at the University of Chicago, the Kavli Foundation, and the Gordon and Betty Moore Foundation. The McGill group acknowledges funding from the Na- tional Sciences and Engineering Research Council of Canada, Canada Research Chairs program, and the Canadian Institute for Advanced Research. R.K. acknowledges support from NASA Hubble Fellowship grant HF-51275.01. B.A.B. is supported by a KICP Fellowship. M.D. acknowledges support from an Alfred P. Sloan Research Fellowship. L.S. acknowledges the support of Yale University and NSF grant AST-1009811. M.M. and L.K. acknowledge the support of NSF grant 0709498. This research used resources of the National Energy Research Scientific Computing Center, which is supported by the Office of Science of the U.S. Department of Energy under Contract No. DE-AC0205CH11231. Some of the results in this paper have been derived using the HEALPix (Górski et al. 2005) package. We acknowledge the use of the Legacy Archive for Microwave Background Data Analysis (LAMBDA). Support for LAMBDA is provided by the NASA Office of Space Science.

\section{REFERENCES}

Addison, G. E., Dunkley, J., Hajian, A., et al. 2012, ApJ, 752, 120

Alam, U., Lukić, Z., \& Bhattacharya, S. 2011, ApJ, 727, 87

Battaglia, N., Bond, J. R., Pfrommer, C., \& Sievers, J. L. 2011a, arXiv: 1109.3709

Battaglia, N., Bond, J. R., Pfrommer, C., \& Sievers, J. L. 2011b, arXiv: 1109.3711

Battaglia, N., Bond, J. R., Pfrommer, C., Sievers, J. L., \& Sijacki, D. 2010, ApJ, 725,91

Béthermin, M., Dole, H., Lagache, G., Le Borgne, D., \& Penin, A. 2011, A\&A, 529, A4

Bode, P., Ostriker, J. P., \& Vikhlinin, A. 2009, ApJ, 700, 989

Bond, J. R., Carr, B. J., \& Hogan, C. J. 1986, ApJ, 306, 428

Bond, J. R., Carr, B. J., \& Hogan, C. J. 1991, ApJ, 367, 420

Carlstrom, J. E., Ade, P. A. R., Aird, K. A., et al. 2011, PASP, 123, 568

Das, S., Marriage, T. A., Ade, P. A. R., et al. 2011, ApJ, 729, 62

de Zotti, G., Ricci, R., Mesa, D., et al. 2005, A\&A, 431, 893

Dunkley, J., Hlozek, R., Sievers, J., et al. 2011, ApJ, 739, 52

Dvorkin, C., \& Hu, W. 2010, Phys. Rev. D, 82, 043513

Dwek, E., \& Arendt, R. G. 1998, ApJ, 508, L9

Fendt, W. A., \& Wandelt, B. D. 2007a, arXiv:0712.0194

Fendt, W. A., \& Wandelt, B. D. 2007b, ApJ, 654, 2

Finkbeiner, D. P., Davis, M., \& Schlegel, D. J. 1999, ApJ, 524, 867

Fixsen, D. J., Dwek, E., Mather, J. C., Bennett, C. L., \& Shafer, R. A. 1998, ApJ, 508,123

Fowler, J. W., Niemack, M. D., Dicker, S. R., et al. 2007, Appl. Opt., 46, 3444

Giavalisco, M., Steidel, C. C., Adelberger, K. L., et al. 1998, ApJ, 503, 543

Gispert, R., Lagache, G., \& Puget, J. L. 2000, A\&A, 360, 1

Górski, K. M., Hivon, E., Banday, A. J., et al. 2005, ApJ, 622, 759

Gruzinov, A., \& Hu, W. 1998, ApJ, 508, 435

Hall, N. R., Keisler, R., Knox, L., et al. 2010, ApJ, 718, 632

Hivon, E., Górski, K. M., Netterfield, C. B., et al. 2002, ApJ, 567, 2

Keisler, R., Reichardt, C. L., Aird, K. A., et al. 2011, ApJ, 743, 28

Knox, L., Cooray, A., Eisenstein, D., \& Haiman, Z. 2001, ApJ, 550, 7

Knox, L., Scoccimarro, R., \& Dodelson, S. 1998, Phys. Rev. Lett., 81, 2004

Komatsu, E., \& Seljak, U. 2002, MNRAS, 336, 1256

Komatsu, E., Smith, K. M., Dunkley, J., et al. 2011, ApJS, 192, 18

Lagache, G., Puget, J.-L., \& Dole, H. 2005, ARA\&A, 43, 727

Larson, D., Dunkley, J., Hinshaw, G., et al. 2011, ApJS, 192, 16

Lau, E. T., Kravtsov, A. V., \& Nagai, D. 2009, ApJ, 705, 1129

Lewis, A., \& Bridle, S. 2002, Phys. Rev. D, 66, 103511

Lewis, A., Challinor, A., \& Lasenby, A. 2000, ApJ, 538, 473

Lueker, M., Reichardt, C. L., Schaffer, K. K., et al. 2010, ApJ, 719, 1045

Mantz, A., Allen, S. W., Ebeling, H., Rapetti, D., \& Drlica-Wagner, A. 2010, MNRAS, 406, 1773

Marsden, G., Ade, P. A. R., Bock, J. J., et al. 2009, ApJ, 707, 1729

Millea, M., Doré, O., Dudley, J., et al. 2012, ApJ, 746, 4

Mauch, T., Murphy, T., Buttery, H. J., et al. 2003, MNRAS, 342, 1117

Negrello, M., Perrotta, F., González-Nuevo, J., et al. 2007, MNRAS, 377, 1557

Padin, S., Staniszewski, Z., Keisler, R., et al. 2008, Appl. Opt., 47, 4418

Parrish, I. J., McCourt, M., Quataert, E., \& Sharma, P. 2012, MNRAS, 419, L29

Percival, W. J., Reid, B. A., Eisenstein, D. J., et al. 2010, MNRAS, 401, 2148

Planck Collaboration, et al. 2011, A\&A, 536, A18

Polenta, G., Marinucci, D., Balbi, A., et al. 2005, J. Cosmol. Astropart. Phys., JCAP11(2005)001 
Reichardt, C. L., Ade, P. A. R., Bock, J. J., et al. 2009, ApJ, 694, 1200

Riess, A. G., Macri, L., Casertano, S., et al. 2011, ApJ, 730, 119

Rozo, E., Wechsler, R. H., Rykoff, E. S., et al. 2010, ApJ, 708, 645

Ruhl, J., Ade, P. A. R., Carlstrom, J. E., et al. 2004, Proc. SPIE, 5498, 11

Scott, D., \& White, M. 1999, A\&A, 346, 1

Sehgal, N., Bode, P., Das, S., et al. 2010, ApJ, 709, 920

Sehgal, N., Trac, H., Acquaviva, V., et al. 2011, ApJ, 732, 44

Sharp, M. K., Marrone, D. P., Carlstrom, J. E., et al. 2010, ApJ, 713, 82

Shaw, L. D., Nagai, D., Bhattacharya, S., \& Lau, E. T. 2010, ApJ, 725, 1452

Shaw, L. D., Rudd, D. H., \& Nagai, D. 2011, ApJ, in press (arXiv:1109.0553)

Shaw, L. D., Zahn, O., Holder, G. P., \& Doré, O. 2009, ApJ, 702, 368

Shirokoff, E., Reichardt, C. L., Shaw, L., et al. 2011, ApJ, 736, 61
Sunyaev, R. A., \& Zel'dovich, Y. B. 1972, Comments Astrophys. Space Phys., 4,173

Sunyaev, R. A., \& Zeldovich, Y. B. 1980, MNRAS, 190, 413

Trac, H., Bode, P., \& Ostriker, J. P. 2011, ApJ, 727, 94

Tristram, M., Macías-Pérez, J. F., Renault, C., \& Santos, D. 2005, MNRAS, 358,833

Vanderlinde, K., Crawford, T. M., de Haan, T., et al. 2010, ApJ, 722, 1180

Vieira, J. D., Crawford, T. M., Switzer, E. R., et al. 2010, ApJ, 719, 763

Viero, M. P., Ade, P. A. R., Bock, J. J., et al. 2009, ApJ, 707, 1766

Vikhlinin, A., Kravtsov, A. V., Burenin, R. A., et al. 2009, ApJ, 692, 1060

Zahn, O., Reichardt, C. L., Shaw, L. D., et al. 2011, arXiv:1111.6386

Zahn, O., Zaldarriaga, M., Hernquist, L., \& McQuinn, M. 2005, ApJ, 630, 657 